\title{
BCL-2 antagonism sensitizes cytotoxic T cell-resistant HIV reservoirs to elimination ex vivo
}

\author{
Yanqin Ren, ${ }^{1}$ Szu Han Huang, ${ }^{1}$ Shabnum Patel, ${ }^{2,3}$ Winiffer D. Conce Alberto, ${ }^{1}$ Dean Magat, ${ }^{1}$ Dughan Ahimovic, ${ }^{1}$ \\ Amanda B. Macedo, ${ }^{3}$ Ryan Durga, ${ }^{3}$ Dora Chan, ${ }^{3}$ Elizabeth Zale, ${ }^{1}$ Talia M. Mota, ${ }^{1}$ Ronald Truong, ${ }^{3}$ Thomas Rohwetter, ${ }^{3}$ \\ Chase D. McCann, ${ }^{1}$ Colin M. Kovacs, ${ }^{4}$ Erika Benko, ${ }^{4}$ Avery Wimpelberg, ${ }^{5}$ Christopher Cannon, ${ }^{5}$ W. David Hardy, ${ }^{5,6}$ Alberto Bosque, ${ }^{3}$ \\ Catherine M. Bollard, ${ }^{2,3}$ and R. Brad Jones ${ }^{1,3}$ \\ 'Division of Infectious Diseases, Department of Medicine, Weill Cornell Medicine, New York, New York, USA. ²enter for Cancer and Immunology Research, Children's National Health System, Washington, DC, \\ USA. ${ }^{3}$ Department of Microbiology, Immunology, and Tropical Medicine, George Washington University, Washington, DC, USA. ${ }^{4}$ Maple Leaf Medical Clinic, Toronto, Ontario, Canada. ${ }^{5}$ Whitman-Walker Health, \\ Washington, DC, USA. 'Division of Infectious Diseases, Department of Medicine, Johns Hopkins University School of Medicine, Baltimore, Maryland, USA.
}

\begin{abstract}
Curing HIV infection will require the elimination of a reservoir of infected CD4 ${ }^{+} \mathrm{T}$ cells that persists despite HIV-specific cytotoxic T cell (CTL) responses. Although viral latency is a critical factor in this persistence, recent evidence also suggests a role for intrinsic resistance of reservoir-harboring cells to CTL killing. This resistance may have contributed to negative outcomes of clinical trials, where pharmacologic latency reversal has thus far failed to drive reductions in HIV reservoirs. Through transcriptional profiling, we herein identified overexpression of the prosurvival factor B cell lymphoma 2 (BCL-2) as a distinguishing feature of $\mathrm{CD} 4^{+} \mathrm{T}$ cells that survived $\mathrm{CTL}$ killing. We show that the inducible HIV reservoir was disproportionately present in BCL- ${ }^{\text {hi }}$ subsets in ex vivo CD4+ $T$ cells. Treatment with the BCL-2 antagonist ABT-199 was not sufficient to drive reductions in ex vivo viral reservoirs when tested either alone or with a latency-reversing agent (LRA). However, the triple combination of strong LRAs, HIV-specific T cells, and a BCL-2 antagonist uniquely enabled the depletion of ex vivo viral reservoirs. Our results provide rationale for novel therapeutic approaches targeting HIV cure and, more generally, suggest consideration of BCL-2 antagonism as a means of enhancing CTL immunotherapy in other settings, such as cancer.
\end{abstract}

\section{Introduction}

In the absence of antiretroviral treatment (ART), HIV maintains sustained viremia in most individuals, resulting in progression to AIDS. Several lines of evidence have established a role for CD8 ${ }^{+}$ $\mathrm{T}$ cells in partially controlling viral replication and delaying this progression (1-5). Although a number of mechanisms contribute to this $(6,7)$, a key mode of action is the direct recognition and elimination of infected cells by $\mathrm{CD}^{+}$cytotoxic T cells (CTLs) (8, 9). Despite this antiviral activity, CTLs are not able to clear all HIV-infected cells from an individual, even when viral replication is abrogated by ART. This is generally attributed to viral latency, which leaves reservoirs of infected cells that invariably reestablish systemic viremia if ART is ever interrupted (10-12).

The "kick-and-kill" (or "shock-and-kill") paradigm proposes to combine latency-reversing agents (LRA) to induce HIV antigen expression with immune effectors, such as CTLs, to eliminate infected cells from the reservoir $(13,14)$. Although kick-and-kill approaches have proven effective in vitro against primary cell models of latency, they have thus far failed to drive measurable

Authorship note: YR and SHH are co-first authors.

Conflict of interest: RBJ has received payments for his role on the scientific advisory board of AbbVie Inc.

Copyright: ( 2020 , American Society for Clinical Investigation.

Submitted: August 6, 2019; Accepted: January 29, 2020; Published: April 13, 2020.

Reference information: / Clin Invest. 2020;130(5):2542-2559.

https://doi.org/10.1172/JCl132374. reductions in frequencies of infected cells in clinical trials (15-20). In an effort to bridge these contrasting results, we have focused on evaluating kick-and-kill approaches against $\mathrm{CD} 4^{+} \mathrm{T}$ cells derived directly ex vivo from ART-suppressed individuals. We previously reported that we were unable to drive reductions in viral reservoirs from these samples, as measured by quantitative viral outgrowth assays (QVOAs), despite the use of potent LRAs and functional CTLs targeting nonescaped viral epitopes (21). In those experiments, we recovered virus from QVOA wells, superinfected autologous $\mathrm{CD}^{+} \mathrm{T}$ cells, and demonstrated that the same CTLs that had been unable to eliminate the latent reservoir efficiently eliminated cells newly infected with these reservoir viruses. These results argued against viral escape or CTL dysfunction as mechanisms by which these reservoirs were not eliminated ex vivo and led us to propose that reservoir-harboring cells from ARTsuppressed individuals are resistant to elimination by CTLs (22). Of note, a separate study has recently shown that virus derived from clonally expanded HIV-infected cells from antiretroviraltreated (ARV-treated) individuals often remains sensitive to autologous CTL, further arguing against epitope escape as a dominant mechanism underlying the persistence of these cells (23).

Natural heterogeneity is known to exist in the intrinsic susceptibility of $\mathrm{CD}^{+} \mathrm{T}$ cells to killing by CTL, supporting the plausibility of a reservoir that has been selected to be CTL resistant. For example, central memory $\mathrm{CD} 44^{+} \mathrm{T}$ cells (TCM cells) are more resistant than transitional $\mathrm{T}$ cell and effector memory T cell (TEM) subsets, and activated $\mathrm{CD} 4^{+} \mathrm{T}$ cells are more susceptible than their resting counterparts 
(24). One study has also reported that $\mathrm{CD}^{+} \mathrm{T}$ cells from $\mathrm{HIV}^{+}$individuals who exhibit natural control of viral replication are intrinsically more sensitive to killing than those from individuals with progressive disease, suggesting a role for the susceptibility of these cells to CTL killing in disease outcome (25). The mechanism for these differential susceptibilities of $\mathrm{CD}^{+}{ }^{+} \mathrm{T}$ cell subsets to CTL-mediated elimination is unclear, though multiple mechanisms of resistance have been identified in other cell types (26-29).

To address the challenge of CTL resistance, we performed RNA-Seq transcriptional profiling of peptide-pulsed primary $\mathrm{CD}^{+} \mathrm{T}$ cells that preferentially survived coculture with corresponding HIV-specific CTL. We identified a number of genes and pathways that were differentially regulated in survivors, including overexpression of the prosurvival factor B cell lymphoma 2 (BCL-2), which we selected for further study. CTL-mediated elimination of target cells occurs when the TCR binds its cognate peptide/MHC-I complex, triggering the release of perforin/granzymes, or through Fas/FasL interactions (30). BCL-2 is a master regulator of apoptosis that can inhibit both the perforin/granzyme B and FasL/Fas pathways by sequestering Bid, thus preventing mitochondrial membrane permeabilization by truncated Bid (30-34). We show that cells harboring the inducible HIV reservoir express high levels of BCL-2 following ex vivo reactivation. In the oncology setting, the prosurvival BCL-2 family proteins have been identified as key factors in the resistance of many tumor cells to death (35-38). BCL-2 antagonists, such as ABT-199 (venetoclax), have been developed as cancer therapies that aim to directly promote the apoptosis of tumor cells, which often overexpress BCL-2 (35-40). By adding ABT-199 to our kick-and-kill cocultures, we were able to achieve the reductions in ex vivo HIV reservoirs that we have been unable to achieve with CTL and LRAs alone. This has direct implications for efforts to eliminate persistent HIV reservoirs and may contribute to our understanding of potential CTL-dependent mechanisms of action of BCL-2 antagonists in other settings, such as cancer.

\section{Results}

Transcriptional profiling of target $C D 4^{+} T$ cells that survive CTL coculture reveals candidate mechanisms of resistance. To identify candidate mechanisms that may confer CTL resistance to HIV reservoir-harboring cells, we first studied differential intrinsic sensitivities to CTL killing in primary $\mathrm{CD} 4^{+} \mathrm{T}$ cells. Given that different maturational phenotypes of $\mathrm{CD} 4^{+} \mathrm{T}$ cells are associated with differential susceptibilities to CTL (24), we sought to minimize this variable by synchronizing target cells in a TCM cell phenotype, as these cells preferentially harbor the latent reservoir (41). This was achieved following the protocol used to generate cells for the cultured TCM model of HIV latency (see Supplemental Methods; supplemental material available online with this article; https://doi.org/10.1172/JCI132374DS1) (42, 43). TCM cells were divided into either a "real" condition, in which half of the cells were labeled with CFSE and pulsed with the HIV-Env peptide RLRDLLLIVTR (RR11), while the other half received no peptide and were labeled with cell-track far-red (CTFR), or a "mock" condition, in which cells were similarly labeled but received no peptide. Both conditions were then cocultured with the corresponding CTL clone (Figure 1A).
This design allowed the isolation of transcriptional profiles associated with preferential survival from profiles that resulted from exposure to an environment containing activated CTL, i.e., (a) the "mock bystanders" and "mock survivors" should not differ from each other, (b) the difference between either mock bystanders or mock survivors and "real bystanders" should reflect exposure of the latter to peptide-stimulated CTL (e.g., cytokine signaling), (c) the difference between real bystanders and "real survivors" should reflect selection for factors that confer CTL resistance, and (d) the difference between real survivors and either of the mock conditions should reflect a combination of $b$ and $\mathrm{c}$ (Figure 1A). Following an overnight coculture, CD $4^{+} \mathrm{T}$ cells under both conditions were sorted into bystander (CTFR) and survivor (CFSE) populations by flow cytometry and subjected to transcriptional profiling by RNA-Seq.

Principal component analysis (PCA) of the resulting RNA-Seq data revealed a pattern that was consistent with the above expectations, with the mock bystanders and mock survivors clustering together, while the real survivors and real bystanders formed distinct clusters (Figure 1B). As expected, the differences between the real bystanders and the mock bystander conditions were predominately attributable to the former having been cocultured with peptide-stimulated CTL, e.g., cytokine signaling, IFN signaling, and $\mathrm{T}$ cell activation (Supplemental Figure 1). Of greater importance to the current study, the comparison between the real survivors and real bystanders identified 1061 differentially expressed genes (DEGs) (FDR < 0.05: 743 upregulated and 318 downregulated). Ingenuity Pathway Analysis (IPA) was performed, and the significantly enriched pathways are shown in Figure 1C (BenjaminiHochberg multiple testing correction, $P<0.05)$. A number of individual genes appeared multiple times in these pathways, as indicated in Figure 1D. To further identify key genes and establish connections between these, we generated gene network diagrams based on the Ingenuity Pathway Knowledge Base. Among these networks, we highlight one that contains components of the following canonical pathways relevant to our hypothesis: CTLmediated apoptosis of target cells, death receptor signaling, IFN signaling, and mitochondrial dysfunction (Figure 1E). This "network 6" and all other networks are listed in Supplemental Table 1, along with scores. Following from this result, we assessed the expression levels of the genes implicated in the CTL-mediated apoptosis of target cells pathway (caspase-2 and BCL-2), as well as poly (ADP-ribose) polymerase (PARP), a mediator of apoptosis that is downstream of caspase activation. We observed expression profiles that were consistent with specific selection of overexpression of BCL-2 and underexpression of caspase-2 and PARP in the real survivor cells that resisted elimination by CTL (Figure $1 \mathrm{~F})$. These results confirm that heterogeneity exists in the intrinsic sensitivity of $\mathrm{CD} 4^{+} \mathrm{T}$ cells to elimination by CTL and is associated with a transcriptional signature implicating multiple gene pathways. We prioritized BCL-2 for validation and further study based on its central position within the network shown in Figure 1E, its central role in cell survival, and its potential to directly antagonize killing by CTL (30-34).

$H I V$-specific CTLs preferentially kill BCL-2 ${ }^{\text {lo }}$ primary $C D 4^{+} T$ cells, thus selecting for $B C L-2^{h i}$ survivors in vitro. We next determined whether the overexpression of BCL-2 transcripts observed 
A

'Real' condition

50/50 ratio of:

$\mathrm{CFSE}^{+}$HIV peptide-labeled targets

$\mathrm{CTFR}^{+}$Unlabeled targets

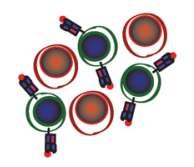

Pulse only CFSE ${ }^{+}$ cells with peptide :

\begin{abstract}
targets
\end{abstract}
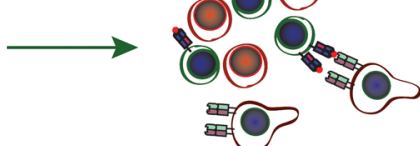
料

Mix both conditions with HIV-specific cytotoxic T-cells

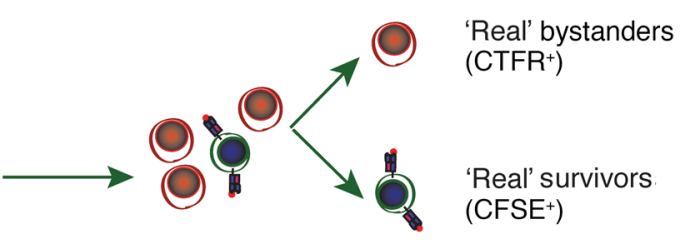

Sort based on CFSE and CTFR by flow cytometry

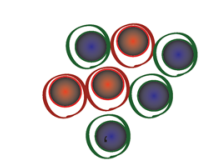

'Mock' condition 50/50 ratio of:
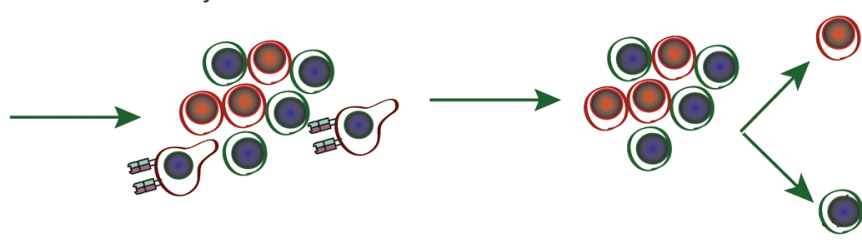

'Mock' bystanders $\left(\mathrm{CTFR}^{+}\right)$

'Mock' survivors (CFSE $\left.{ }^{+}\right)$

B

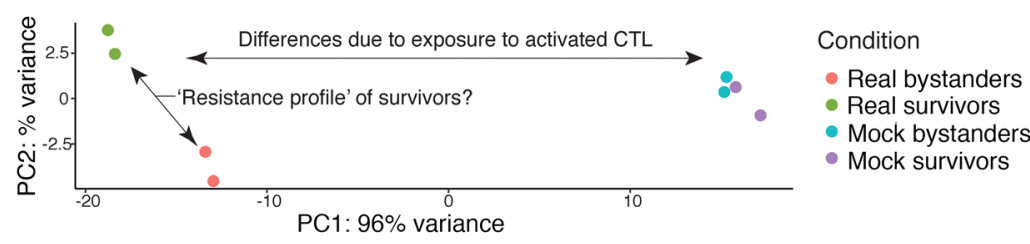

D

\begin{tabular}{cc}
\hline Gene name & Count \\
\hline RALA & 8 \\
RALB & 7 \\
RAP1A & 7 \\
BC L2 & 5 \\
PPP2R5D & 4 \\
RPS27A & 4 \\
\hline
\end{tabular}

\section{C}

- Positive $Z$ score $\square Z$ score $=0$

negative $Z$ score $\square$ No activity pattern available

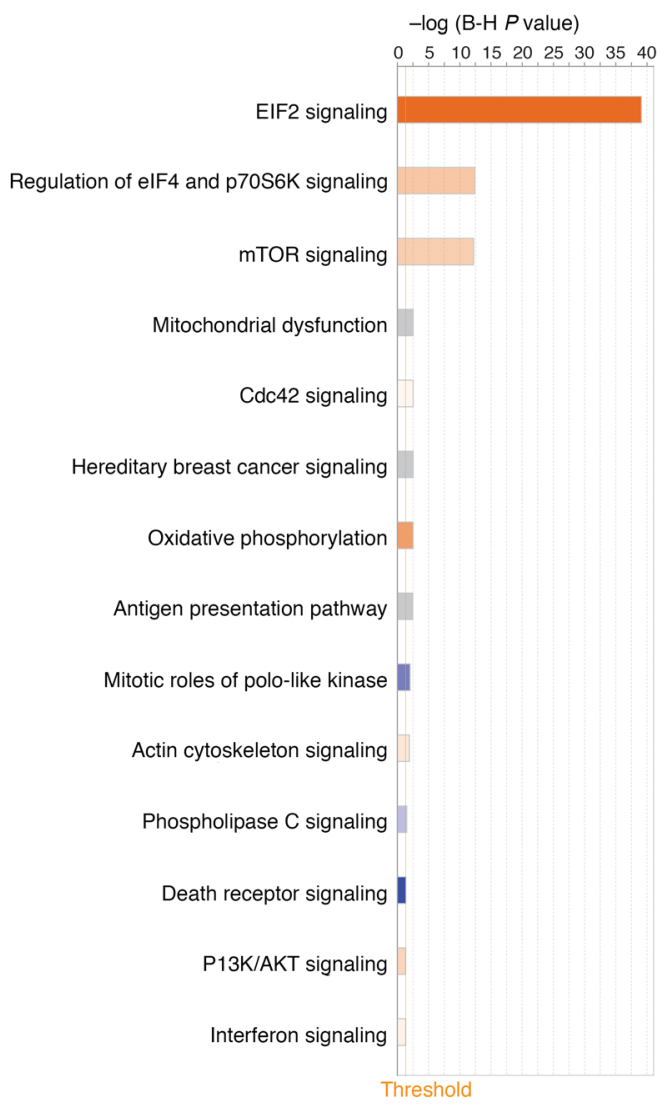

E

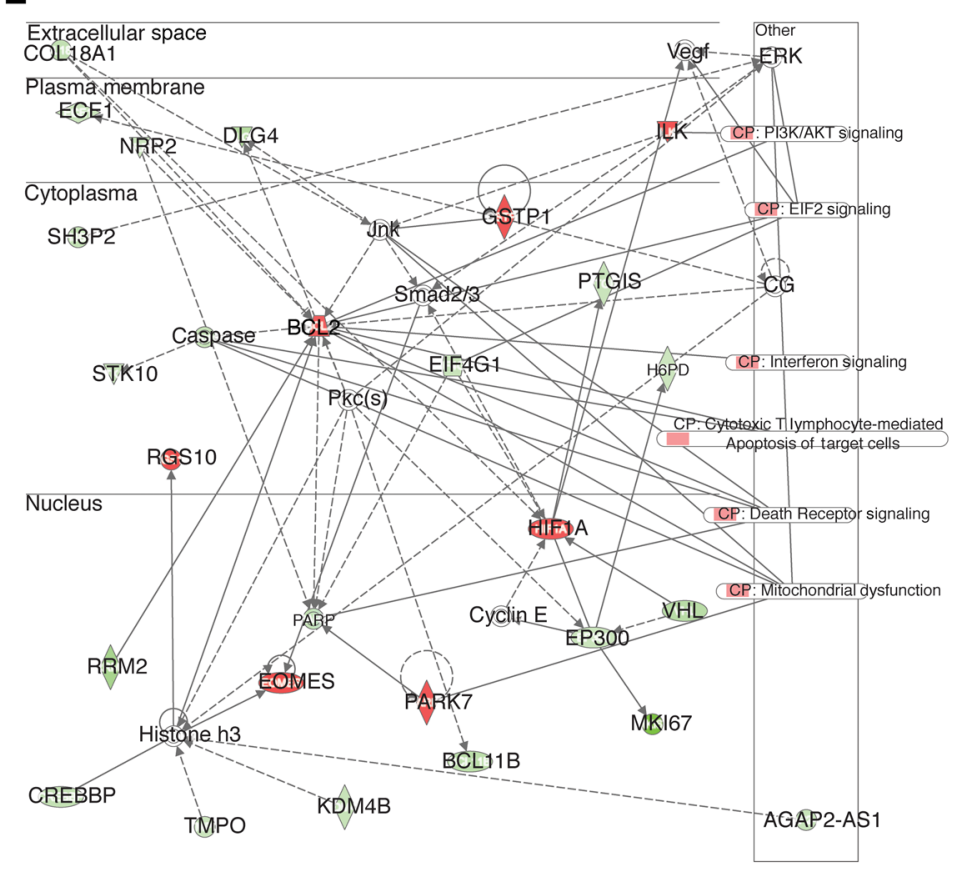

$\mathbf{F}$
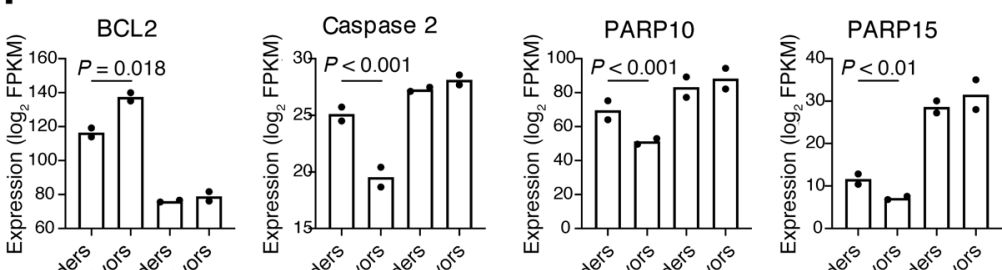
Figure 1. Transcriptional profiling of target $\mathrm{CD4}^{+} \mathrm{T}$ cells that survive $\mathrm{CTL}$ coculture reveals candidate mechanisms of resistance. (A) Schematic of peptide-pulse killing assay and flow sorting for transcriptional profiling. (B) PCA showing clustering of cell populations, as indicated. (C) IPA results showing the pathways that were significantly enriched between real bystanders and real survivors. Orange bars, positive $Z$ scores; blue bars, negative $Z$ scores; gray bars, no activity pattern. (D) Top 6 genes by numbers of instances in significant pathways from C. (E) IPA network analysis (subcellular display) showing a significantly enriched network. Interactions with significant pathways from C and with CTL-mediated apoptosis of target cells are also shown. Red shading indicates overexpression in real survivors, and green indicates underexpression, both in comparison with real bystanders. (F) BCL-2 as well as upstream (CASP2) and downstream (PARP) gene expression levels in all 4 conditions. Shown are fragments per kilobase of exon model per million mapped reads (FPKM) from RNA-Seq. FDR-adjusted $P$ values from DESeq analysis are shown.

in RNA-Seq data was reflected at the protein level, with the hypothesis that BCL- ${ }^{\text {hi }} \mathrm{CD} 4^{+} \mathrm{T}$ cells would preferentially survive CTL-mediated killing. We tested this by coculturing HIV-specific CTLs with autologous $\mathrm{CD}^{+} \mathrm{T}$ cells that had been pulsed with various concentrations of its cognate peptide and measuring BCL-2 expression levels in surviving CD $4^{+} \mathrm{T}$ cells (Figure $2, \mathrm{~A}$ and $\mathrm{B}$ ). We observed significant losses in viable $\mathrm{CD} 4^{+} \mathrm{T}$ cells with increasing peptide concentrations $(10 \mu \mathrm{g} / \mathrm{mL}$ peptide vs. no treatment, 3.2-fold decrease, $P<0.0001 ; 2.1$-fold decrease vs. $0.01 \mu \mathrm{g} / \mathrm{mL}$ peptide, $P<0.0001$; Figure $2 \mathrm{C}$ ) and a corresponding increase in BCL-2 expression in the remaining $\mathrm{CD}^{+} \mathrm{T}$ cells $(10 \mu \mathrm{g} / \mathrm{mL}$ vs. no treatment, $P=0.008$; vs. $1 \mu \mathrm{g} / \mathrm{mL}, P=0.03$; Figure $2, \mathrm{C}$ and $\mathrm{D}$ ). This effect was confirmed by assessing the impact of CTL killing on total numbers of target cells as divided into BCL- $2^{\text {hi }}$ and BCL-2 ${ }^{\text {lo }}$ populations (Figure $2 \mathrm{E}$ ). We observed a progressive decrease in the numbers of BCL-2 $2^{\text {lo }}$ cells with increasing peptide concentrations (mean count 83,000 at $0.01 \mu \mathrm{g} / \mathrm{mL}$ peptide vs. 45,000 at $10 \mu \mathrm{g} / \mathrm{mL}$ peptide, $P=0.009$; Figure $2 \mathrm{E}$ ), alongside a lack of significant change in the numbers of BCL-2 ${ }^{\text {hi }}$ cells, even at $10 \mu \mathrm{g} / \mathrm{mL}$ of RR11 peptide (Figure $2 \mathrm{E}$ ). Thus, these data support that the natural heterogeneity of BCL-2 expression within ex vivo $\mathrm{CD}^{+} \mathrm{T}$ cells is sufficient to influence susceptibility to CTL killing, with BCL-2 ${ }^{\text {hi }}$ cells exhibiting preferential survival. This association could either reflect differences across maturational phenotypes - where, for example, naive $\mathrm{CD} 4^{+} \mathrm{T}$ cells may both express lower levels of BCL-2 and be more susceptible to CTL killing - or may also reflect heterogeneity of these parameters within a given phenotype. To distinguish between these, we performed a killing assay similar to that shown in Figure 2E, with the addition of phenotypic marker staining to discriminate naive $\left(\mathrm{CD} 45 \mathrm{RA}^{+} \mathrm{C}-\right.$ $\mathrm{CR7}^{+}$), TCM (CD45RA-CCR7 ${ }^{+}$), and TEM (CD45RA'CCR7-) populations. Parallel experiments were performed where cells were either activated with anti-CD3/anti-CD28 before peptide pulsing and coculture or were peptide pulsed without prior activation. We observed the preferential survival of BCL-2 ${ }^{\text {hi }}$ cells within each of these populations, whether or not cells had been activated (Figure $2 \mathrm{~F}$ ). The most pronounced skewing in BCL-2 expression was observed within the TEM cells (Figure 2F), which corresponded with a greater degree of killing of this population (percentage killed at $5 \mu \mathrm{g} / \mathrm{mL}$ peptide by phenotype: TCM cells, $21.7 \%$; TEM cells, $63.9 \%$; naive cells, $36.8 \%$ ). Thus, even within a given matu- rational population, the relative expression of BCL-2 is associated with susceptibility to elimination by CTL.

The reactivatable HIV reservoir is preferentially harbored in $B C L-2^{\text {hi }}$ cells in individuals on long-term ART. Given the above observations that BCL- $2^{\text {hi }}$ cells preferentially resist killing by CTL, we next probed a potential role for this mechanism in the persistence of the HIV reservoir. We first assessed BCL-2 expression levels in ex vivo $\mathrm{CD} 4^{+} \mathrm{T}$ cells from ART-suppressed donors, following latency reversal with PMA/ionomycin (PMA/I) (Figure 3A). This was accomplished using a recently developed flow cytometry technique that allows for the identification and phenotypic characterization of this extremely rare population (44). We established a gating strategy using cells from an ART-naive, chronically $\mathrm{HIV}^{+}$ individual (OM5374) and an HIV donor (OM6960) (Figure 3A). As expected, we observed a lack of $\mathrm{Gag}^{+}$cells in the $\mathrm{HIV}^{-}$sample, contrasted by a detectable population in the $\mathrm{HIV}^{+}$sample, which was enhanced by PMA/I stimulation.

We extended this assay to measure differences of BCL-2 expression levels between HIV-infected and uninfected cells among ex vivo $\mathrm{CD}^{+} \mathrm{T}$ cells from 6 additional durably ART-suppressed study participants (Table 1). We observed extremely rare HIV-infected populations from each participant following PMA/I stimulation, while no $\mathrm{Gag}^{+}$events were observed in unstimulated cells (Figure $3 \mathrm{~B})$. For each individual, $\mathrm{HIV}-\mathrm{Gag}^{+}$populations were found to express higher levels of BCL-2 (MFI 2874, range: 1460-5820) than corresponding Gag- populations (MFI 1215, range: 1100-1320) ( $P$ $=0.01$, Figure $3, \mathrm{C}$ and D). In contrast to these ART-suppressed individuals, we observed similar BCL-2 expression levels between the Gag+ (MFI mean: 1001, range: 965-1450) and Gag- cell populations (MFI mean: 1007, range: 1093-1350) from 4 ART-naive participants (Table 2) following PMA/I stimulation (Figure 3, E and F). Although we acknowledge inherent limitations of analyzing such very rare events, we draw confidence in our conclusion from the observation that this difference was statistically robust across a cohort of 6 individuals $(P=0.016$; Figure 3D). Thus, we observed that, following reactivation, the HIV reservoir in ART-suppressed individuals capable of producing Gag is preferentially present in BCL- $2^{\text {hi }}$ cells. This suggests that the levels of BCL-2 overexpression from ARV-treated individuals are not simply the result of HIV expression, but rather may be a feature that is enriched in cells making up long-lived HIV reservoirs.

As an additional method for assessing the BCL-2 expression profile of reservoir-harboring cells, we sorted ex vivo CD4 ${ }^{+}$ $\mathrm{T}$ cells from long-term ART-suppressed individuals into BCL- ${ }^{\text {hi }}$ and BCL-2 ${ }^{\mathrm{lo}}$ populations by flow cytometry and quantified HIV DNA in each population. HIV DNA was measured using a recently developed droplet digital PCR (ddPCR) method that allows for the discrimination of relatively intact proviruses that contain binding sites for both gag and env primer/probe pairs (45). We observed significantly higher frequencies of HIV proviruses in BCL- $2{ }^{\text {hi }} \mathrm{CD} 4^{+}$T cells compared with a BCL- $2^{\text {lo }}$ population $(P=0.02$ for intact and total gag) (Figure 4A). We next determined whether this enrichment of infected cells in the BCL-2 ${ }^{\text {hi }}$ subset was reflective of differences across maturational populations or whether BCL-2 ${ }^{\text {hi }}$ cells would be enriched for infected cells even within a given memory population. We included the maturational markers CCR7 and CD45RA in our flow cytometry panel and sorted cells 


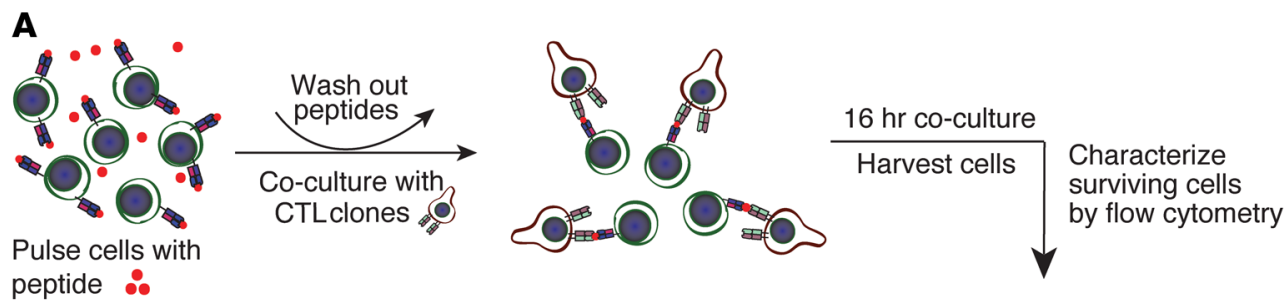

B
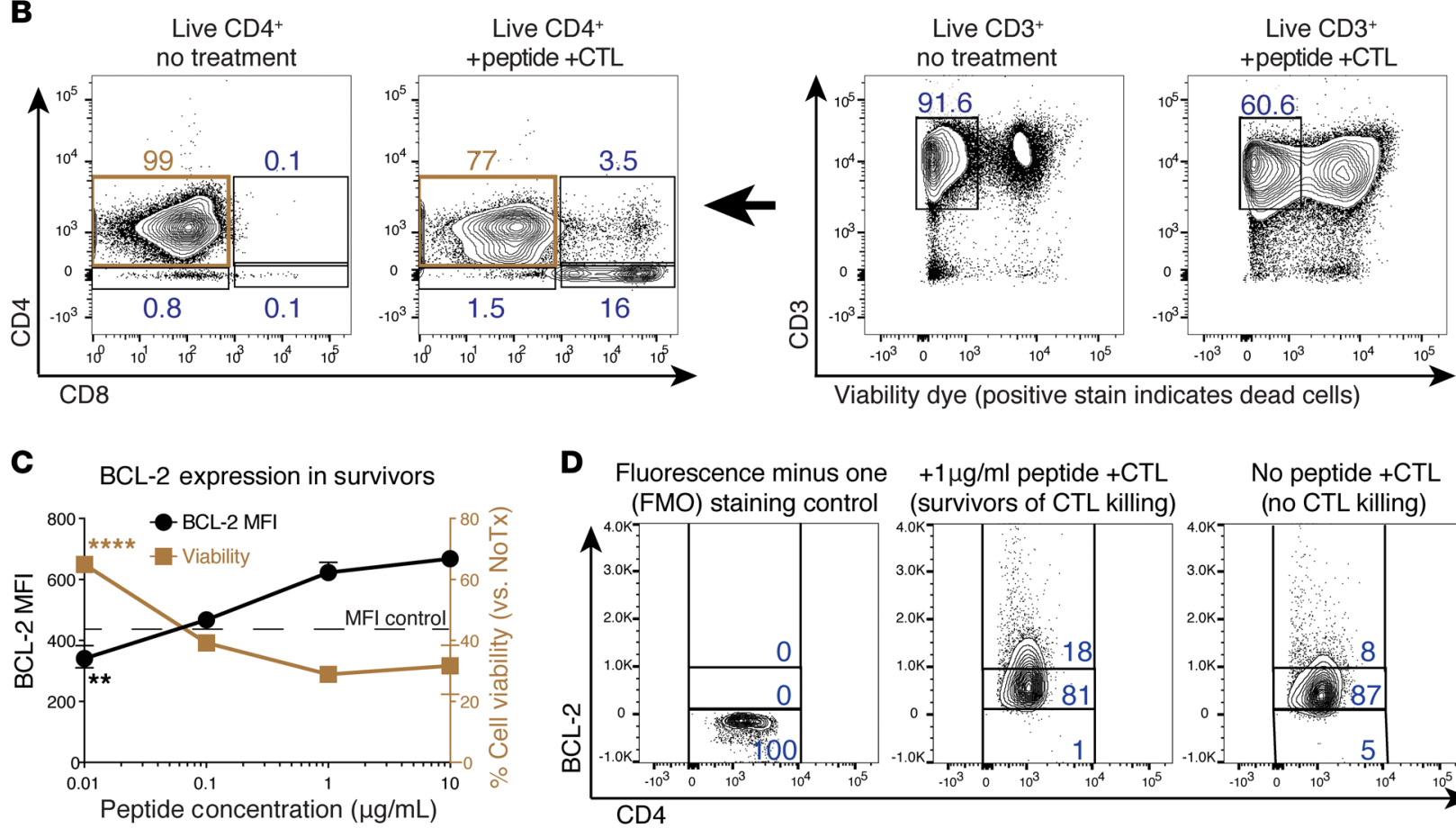

D Fluorescence minus one $+1 \mu \mathrm{g} / \mathrm{ml}$ peptide $+\mathrm{CTL}$ (FMO) staining control (survivors of CTL killing)
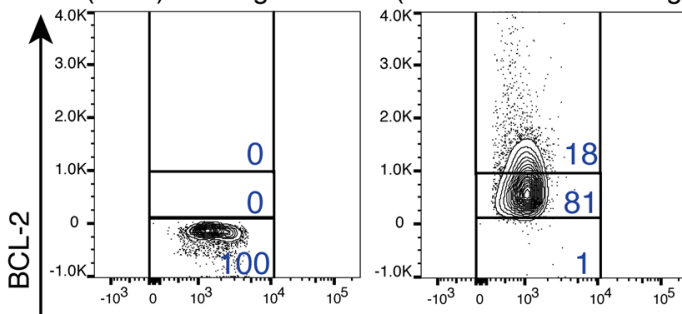

No peptide $+\mathrm{CTL}$
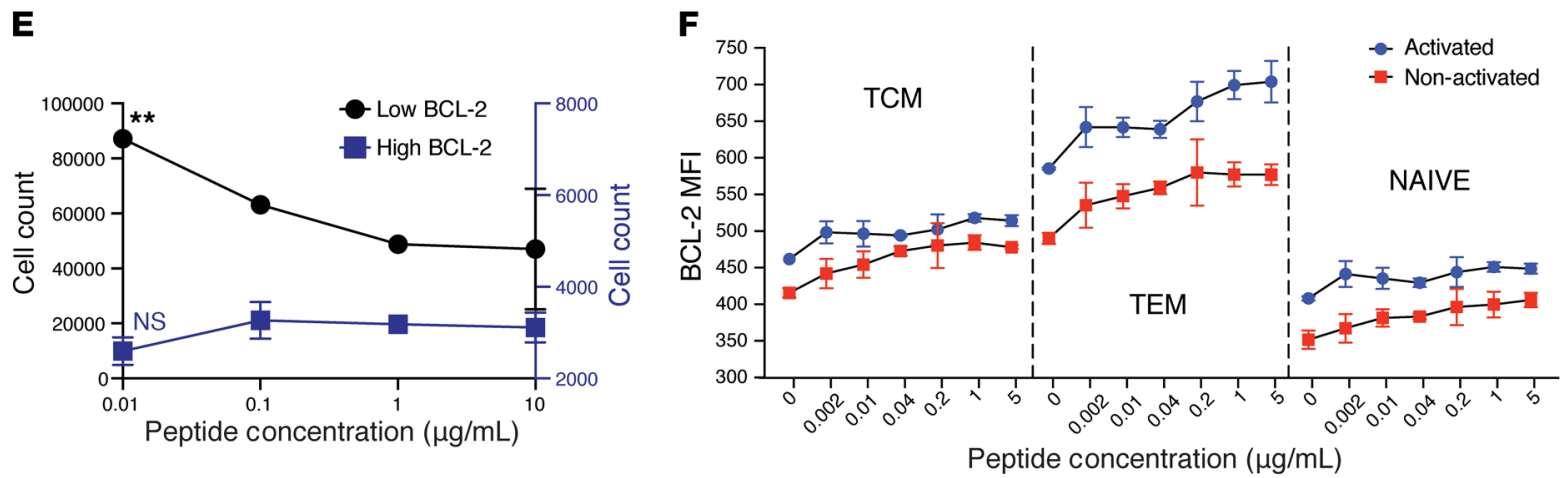

Figure 2. CD8 ${ }^{+}$CTL preferentially eliminate CD4 ${ }^{+}$T cells with low BCL-2 expression levels. (A) Schematic of peptide-pulse and killing assay. (B) Representative gating strategy of flow cytometry plots to identify surviving CD4+ $T$ cells and CD4/CD8 ratios in either no-treatment or + peptide + CTL conditions. (C) Graph of total BCL-2 MFI (left axis, black line) and CD4+ $T$ cell viability normalized to the no-treatment condition (right axis, brown line), following a peptide-pulse killing assay. Total BCL-2 MFI was calculated based on viable CD4+ $T$ cells. The dashed line indicates the BCL-2 MFI of an untreated control. (D) Flow cytometry plots depicting BCL-2 gating strategy for $B C L-2^{\text {hi }}$ and $B C L-2^{10}$ populations. (E) Graph depicting CD4 ${ }^{+} T$ cell counts in $B C L-2^{\text {hi }}($ right axis, blue) and BCL-2 ${ }^{10}$ (left axis, black) populations after CTL killing with different concentration peptide-pulsing treatments. Samples were run in triplicate, and shown are median \pm range. (F) The data shown are analogous to those in C, but with 2 additions: (a) killing assays were performed in parallel on CD4+ cells that had either been activated with anti-CD3/anti-CD28 or were used directly ex vivo (nonactivated); and (b) the markers CD45RA and CCR7 were included in the flow panel to discriminate naive cells (CD45RA+CCR7+), TCM cells (CD45RA-CCR7+), and TEM cells (CD45RA-CCR7-). Statistical significance was determined by $t$ test. ${ }^{* *} P<0.01 ;{ }^{* * *} P<0.0001$.

from 2 donors into BCL-2 $2^{\text {hi }}$ and BCL-2 $2^{\text {lo }}$ subsets for each of naive $\left(\mathrm{CD} 45 \mathrm{RA}^{+} \mathrm{CCR} 7^{+}\right)$, TCM $\left(\mathrm{CD} 45 \mathrm{RA}^{-} \mathrm{CCR} 7^{+}\right)$, and TEM $\left(\mathrm{CD} 45 \mathrm{RA}{ }^{-}\right.$ CCR7-) populations (Figure 4B). For both individuals, we observed pronounced enrichments of intact HIV DNA in the BCL-2 $2^{\text {hi }}$ versus
BCL-2 ${ }^{\text {lo }}$ populations. These patterns were also reflected at the level of total HIV DNA in each memory subset (Figure 4C). These data provide an additional line of evidence supporting the idea that the HIV reservoir is preferentially harbored in BCL- $2^{\text {hi }}$ cells in individ- 
uals on long-term ART and indicate that this is not merely reflective of differences across maturational phenotypes.

BCL-2 antagonist ABT-199 fails to reduce either total HIV DNA or infectious reservoirs from ex vivo $C D 4^{+} T$ cells from $A R V$-treated donors, but can drive reductions in a primary cell latency model. A previous study reported that the combination of ABT-199 with anti-CD3/anti-CD28 antibodies was sufficient to drive reductions in the frequencies of HIV-infected cells taken ex vivo from ART-suppressed participants (46). However, this was not associated with reductions in the amounts of HIV RNA released into culture supernatants, and the effect on the inducible infectious reservoir as measured by QVOA was not tested. As a prelude to assessing the abilities of ABT-199 to sensitize HIV reservoirharboring cells to elimination by CTL, we therefore determined whether this agent had activity against latently HIV-infected cells when used either alone or in combination with an LRA.

In our experiments, the effects of BCL-2 antagonist ABT-199 were assessed using an HIV eradication (HIVE) assay (Figure 5A), where changes in infected cells are measured by both ddPCR to measure total frequencies of infected cells (total HIV DNA) and QVOA to measure replication-competent reservoirs (infectious units). This distinction is important as, in ex vivo $\mathrm{CD} 4^{+} \mathrm{T}$ cells from ARV-treated individuals, the large majority of HIV DNA represents defective proviruses with no potential for viral replication (47). In our HIVE assays, we focused on the protein kinase $\mathrm{C}$ agonist bryostatin- 1 as the LRA, as we had observed that it mitigated the appreciable levels of nonspecific $\mathrm{CD}^{+} \mathrm{T}$ cell toxicity induced by ABT199 (Supplemental Figure 2). Potent activation of CD $4^{+} \mathrm{T}$ cells by bryostatin-1 was confirmed by CD69 staining (Supplemental Figure 3). We were careful to account for cell death in our QVOAs by counting only viable $\mathrm{CD} 4^{+} \mathrm{T}$ cells by flow cytometry following a 24-hour drug washout period to calculate infectious units per million (IUPM) CD4 ${ }^{+} \mathrm{T}$ cells. We further confirmed that prior treatment with ABT-199 did not continue to negatively affect viability after the 24-hour washout, which may have otherwise introduced inaccuracy into our QVOA measurements (Supplemental Figure 4). DNAse I was also included in the HIVE coculture medium to degrade the genomes of killed target cells such that these would not be measured by ddPCR.

We first tested to determine whether ABT-199 would drive reductions when targeting "natural" HIV reservoirs in ex vivo CD $4^{+}$ $\mathrm{T}$ cells from ART-suppressed donors. A representative example of a HIVE assay is shown in Figure 5B. We did not observe reductions in either HIV DNA or IUPM following treatment with ABT-199 (1 $\mu \mathrm{M}$ or $100 \mathrm{nM}$ ), either alone or in combination with bryostatin-1 (Figure 5B). In this initial experiment, the overall loss in viability meant that we had insufficient cells to assess conditions treated solely with ABT-199 $(1 \mu \mathrm{M})$ by QVOA. The only significant differences that we observed were increases in IUPM following treatment with bryostatin- 1 alone $(P<0.001$, Figure $5 \mathrm{~B})$. We extended this HIVE assay to a total of $8 \mathrm{ARV}$-treated donors and consistently observed a lack of significant differences in either HIV DNA or IUPM between untreated conditions and ABT-199 (both $1 \mu \mathrm{M}$ and $100 \mathrm{nM}$ ), tested either alone or in combination with bryostatin-1 (Figure 5, C and D). In contrast, the increases in IUPM observed with bryostatin- 1 treatment were found to be consistent across this population $(P<0.01$ at $1 \mu \mathrm{M}$ and $P=0.03$ at $100 \mathrm{nM}$; Figure 5,
C and D). Thus, the BCL-2 antagonist ABT-199 was not sufficient to drive reductions in ex vivo viral reservoirs - including when combined with the potent LRA bryostatin-1. Although peripheral to the main hypothesis of the current study, we were curious to see whether this combination would be sufficient to drive the elimination of infected cells in a well-characterized primary cell model of HIV latency $(48,49)$. This model typically harbors approximately $1 \%$ latently infected cells that can be reactivated to produce HIV by anti-CD3/anti-CD28. This frequency is much too high to be measured by a typical QVOA, which is designed to detect infected cell frequencies of approximately $0.00001 \%$ to $0.001 \%$. Thus, to enable direct comparison with our results from "natural" HIV reservoirs, we generated target populations with reduced infectedcell frequencies by spiking latency model cells into autologous $\mathrm{CD}^{+} \mathrm{T}$ cells at ratios of 1 model cell:100-1,000 ex vivo CD4 ${ }^{+} \mathrm{T}$ cells (Supplemental Figure 5A). In contrast to natural HIV reservoirs, we observed that ABT-199 $(1 \mu \mathrm{M}$ and $100 \mathrm{nM})$, alone or in combination with bryostatin-1, drove reductions in latency model cells as measured either by ddPCR or QVOA (Supplemental Figure 5). Most strikingly, we observed a 130-fold reduction in IUPM in the bryostatin- $1+1 \mu \mathrm{M}$ ABT-199 condition $(P<0.0001)$ and a 21-fold reduction in IUPM in the bryostatin-1 + $100 \mathrm{nM}$ ABT-199 condition $(P<0.0001$, Supplemental Figure $5 \mathrm{~B})$. Our results are consistent with a previous study that also reported infected-cell reductions in a latency model following treatment with ABT-199 and anti-CD3/anti-CD28 (as an LRA) (46). These spiked latency model HIVEs also offer validation for our treatment conditions and confirm our ability to measure changes in the reservoir in HIVE assays. Thus, our results firmly establish that, while effective against a latency model, ABT-199 in combination with the LRA bryostatin-1 was insufficient to reduce HIV reservoirs ex vivo.

Combinations of a potent LRA, HIV-specific CTL, and a BCL-2 antagonist drive reductions in $H I V$ reservoirs from ex vivo $C D 4^{+} T$ cells. In our hands, both combinations of LRA + ABT-199 and of LRA + CTL were individually effective against primary cell latency models, but not against ex vivo reservoirs. We therefore next tested the central hypothesis of the current study, that a combination of these treatments would deplete ex vivo reservoirs as a result of ABT-199 counteracting resistance to CTL-mediated elimination (Figure 6A). In an initial experiment, using cells from an ARV-treated donor OM5011 (see Table 1), we continued to use bryostatin-1 as an LRA and measured the elimination of latently infected cells with ddPCR, quantifying HIV DNA at both gag and env amplicons. Using 2 different autologous HIV-Gag-specific CTL clones (targeting the ACQGVGGPGHK AK11 and the HPVHAGPIA HA9 epitopes), we observed significant depletions in HIV DNA as measured at the env target sequence uniquely in the triplecombination condition of bryostatin-1 + Gag-specific CTL + ABT199 (both $P<0.01$, respectively; Figure 6B). We included an autologous CMV-pp65-specific CTL clone as an additional control and observed a lack of depletion in HIV DNA, as expected (Figure 6B). Using samples from the same experiment, we observed nonsignificant trends toward depletion in HIV DNA as measured at the gag target sequence for both CTL conditions (AK11, $P=0.20$; HA9, $P=0.15$; Figure 6C). In a second experiment, we used an autologous polyclonal HIV-specific T cell product that has been developed for T cell therapy (termed HSTs) $(50,51)$. The HSTs used in 
A
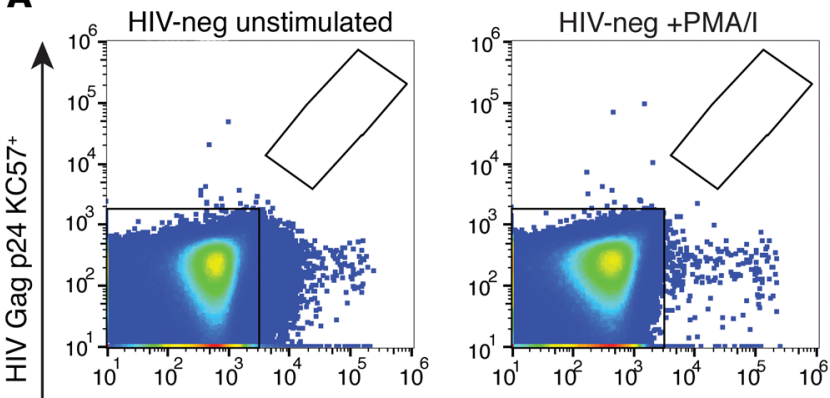

ART-naive donor OM5374 unstimulated

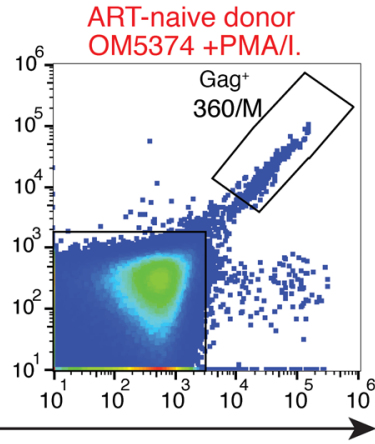

HIV Gag p24 p24.2
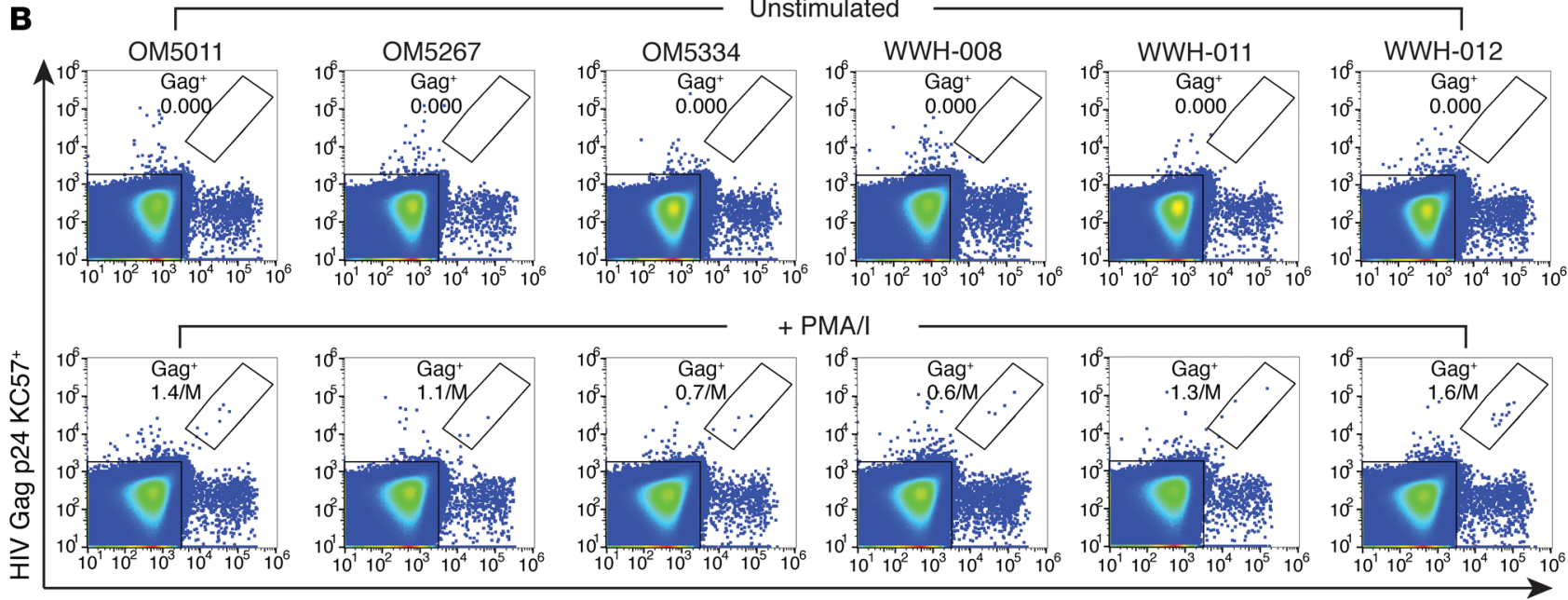

HIV Gag p24 p24.2+
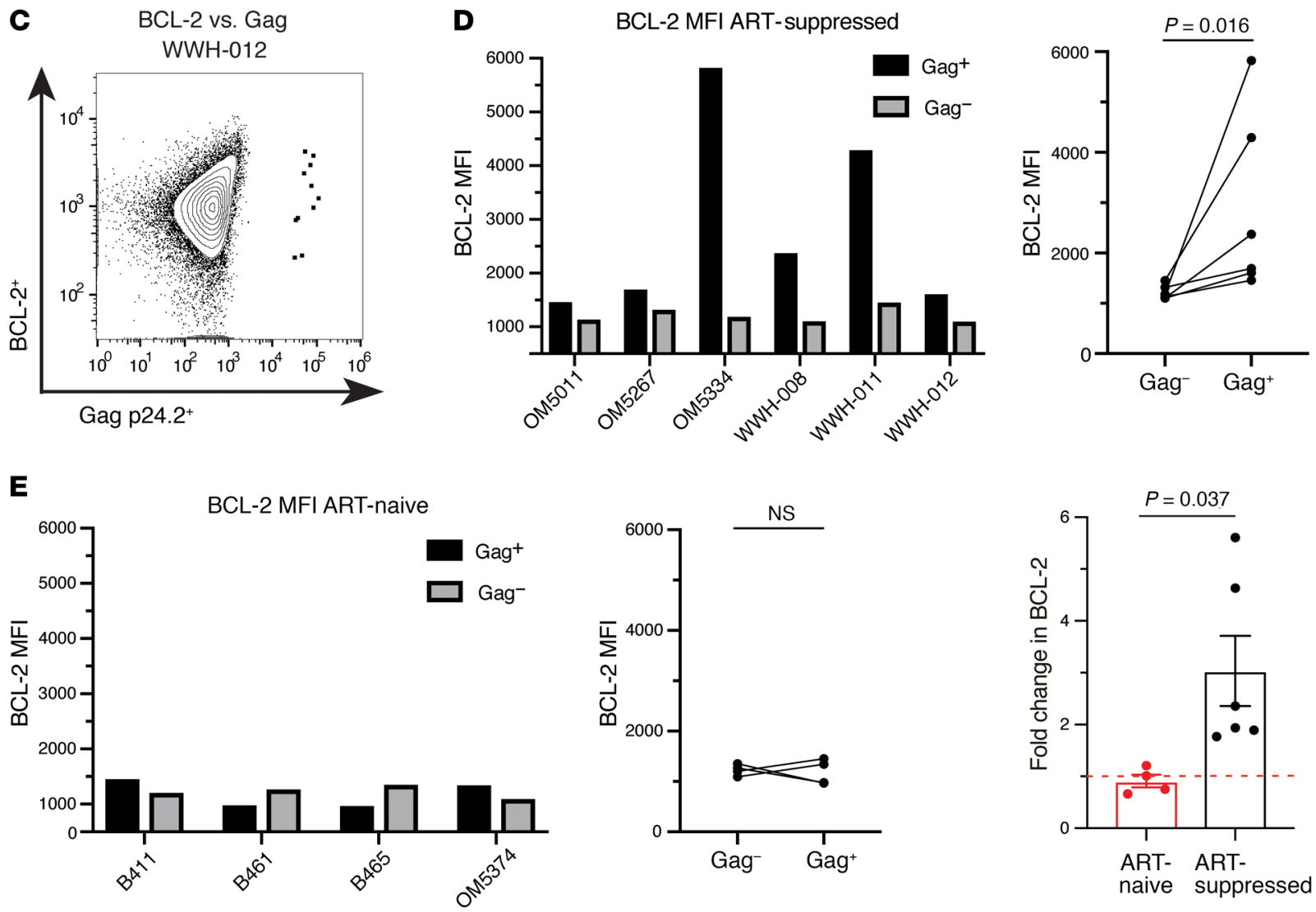
Figure 3. HIV reservoirs are preferentially harbored in BCL-2 ${ }^{\text {hi }}$ expressing $\mathrm{CD4}^{+} \mathrm{T}$ cells following ex vivo reactivation. (A) Flow cytometry plots depicting strategy for identifying HIV-expressing cells by gating on populations that were double-positive for the 2 HIV Gag antibodies. Each plot represents 4-8 $\times 10^{6}$ events. (B) Flow cytometry plots showing HIV-expressing cells from 6 HIV-infected ART-suppressed donors: unstimulated (top row) and stimulated with PMA/I (bottom row). The numbers adjacent to the Gag ${ }^{+}$gates indicate the numbers of events detected per million cells. (C) Flow cytometry plot depicting BCL-2 versus Gag expression in ex vivo $C D 4^{+} T$ cells from an ART-suppressed donor. ( $D$ and $\mathbf{E}$ ) $\mathrm{BCL}-2 \mathrm{MFI}$ of $\mathrm{Gag}^{+}$and Gag populations in ex vivo $\mathrm{CD} 4^{+} \mathrm{T}$ cells from (D) ART-suppressed donors (the same donors as in B) or (E) 4 ARV-naive donors, after PMA/I stimulation (Wilcoxon's signed-rank test). (F) Significantly greater differences in BCL-2 expression, between $\mathrm{Cag}^{+}$and $\mathrm{Cag}$ CD4 ${ }^{+} \mathrm{T}$ cells, were observed in ART-suppressed donors compared with ART-naive individuals (unpaired $t$ test).

this study (Figure 6, D and E, and Figures 7 and 8) exhibited high frequencies of HIV-specific T cells (IFN- $\gamma$ responses to HIV-Gag/ Nef/Pol peptide pools, range: $6970-28,130 \mathrm{SFC} / 10^{6}$ cells); they were confirmed to respond to multiple epitopes by ELISPOT and intracellular cytokine staining (Supplemental Figure 6, A-C) and exhibited strong cytotoxicity against peptide-pulsed cells (Supplemental Figure 6D). In this experiment, we observed that the triple combination of bryostatin-1 + HSTs + ABT-199 uniquely drove a significant reduction in HIV DNA, as measured by primers and probes targeting gag (Figure 6D); however, a trend toward a reduction in ddPCR targeting env did not reach statistical significance $(P$ $=0.06$; Figure $6 \mathrm{E}$ ). Although these initial results did provide some support for our hypothesis, we also considered them to be somewhat marginal due to the lack of consistent statistical significance. Given how resource intensive these HIVE assays are (e.g., using $~ 1$ $\times 10^{9} \mathrm{PBMCs}$ per donor per experiment), we opted to transition to the use of the maximally activating LRA anti-CD3/anti-CD28 for HIVE assays, positing that this would enhance our ability to detect an ability of ABT-199 to sensitive reservoir-harboring cells to elimination by kick-and-kill.

We assessed the combination of anti-CD3/anti-CD28, ABT199, and autologous HSTs in an initial HIVE assay targeting ex vivo
$\mathrm{CD}^{+} \mathrm{T}$ cells from ART-suppressed donor OM5148. The elimination of reservoir-harboring cells was measured in parallel by ddPCR and QVOAs (Figure 7A). We observed significant reductions in total HIV DNA (Figure 7, B and C) following treatment with antiCD3/anti-CD28 and HSTs, as well as with anti-CD3/anti-CD28 and ABT-199, and further significant reductions with anti-CD3/ anti-CD28 + HSTs + ABT-199 (Figure 7B, 3.4-fold vs. no treatment, $P<0.0001 ; 2.1$-fold vs. anti-CD3/anti-CD28 + HSTs, $P=0.009$; 1.9-fold vs. anti-CD3/anti-CD28 + ABT-199, $P=0.03$ ). Consistent with our previous work, the decrease in HIV DNA with a maximal LRA $+\mathrm{T}$ cells was not mirrored by a decrease in levels of replication-competent provirus as measured by QVOAs. Only by combining ABT-199 with anti-CD3/anti-CD28 + HSTs were we able to observe a significant reduction in this infectious reservoir, with no p24 $4^{+}$wells observed in the QVOA (IUPM 0 vs. no treatment: 0.66, $P=0.02$; Figure 7D). We next performed an analogous HIVE using an autologous HIV-specific CTL clone targeting a nonescaped HIV epitope, which had previously failed to eliminate natural HIV reservoirs in the absence of ABT-199 (21). As before, treatment with anti-CD3/anti-CD28 + CTL led to significant 2.1-fold reductions in HIV DNA, while further significant 5.7-fold reductions were observed with anti-CD3/anti-CD28 + CTL clone + ABT-199 (Figure $7 \mathrm{C})$. However, significant decreases in IUPM were uniquely observed with the triple combination of anti-CD3/anti-CD28, CTL clones, and ABT-199 ( 0.32 vs. 2.63 no treatment, $P<0.001$; Figure 7E). Thus, in 2 initial HIVE assays using either HSTs or a CTL clone, we observed that ABT-199 facilitated reductions in IUPM that were not observed with effectors + LRA without ABT-199.

We next tested these treatment conditions in 8 additional HIVE assays, using ex vivo $\mathrm{CD}^{+} \mathrm{T}$ cells from 7 participants. For 3 HST-based HIVE assays, we also ran separate matched HIVE assays using autologous HIV-specific CTL clones confirmed to target nonescaped epitopes (Figure 8); CTL clones, along with HSTs, are collectively referred to hereafter as HIV-specific effectors. As in the above experiments, we observed appreciable nonspecific cell toxicity in the ABT-199-treated conditions. This was accounted for in all HIVE assays by applying only viable $\mathrm{CD} 4^{+} \mathrm{T}$ cell counts when plating QVOAs and calculating IUPM (Supplemental Fig-

Table 1. ART-suppressed participant clinical data

\begin{tabular}{|c|c|c|c|c|c|c|c|}
\hline Participant ID & Sex & Age (yr) & Ethnicity & ART regimen & $\begin{array}{l}\text { Duration of viral load } \\
\text { undetectable (month) }\end{array}$ & $\begin{array}{l}\text { Viral load } \\
\text { (copies } / \mathrm{mL} \text { ) }\end{array}$ & $\begin{array}{l}\text { Estimated time } \\
\text { between infection } \\
\text { and ART (month) }\end{array}$ \\
\hline OM5011 & M & 46 & White & 3TC/ABC/DTG & 133 & $<50$ & 38 \\
\hline 0M5334 & M & 34 & White & Genvoya/edurant & 63 & $<50$ & 2 \\
\hline OM5148 & M & 48 & White & $3 \mathrm{TC} / \mathrm{ABC} / \mathrm{NVP}$ & 149 & $<50$ & 57 \\
\hline 0M5365 & M & 58 & White & $\begin{array}{l}\text { Kivexa/tmc114/rtv/tmc125/ } \\
\text { integrase/maraviroc }\end{array}$ & 114 & $<50$ & 18 \\
\hline 0M5220 & M & 54 & White & ЗТC/ABC/DTG & 102 & $<50$ & Unknown \\
\hline WWH-B004 & $\mathrm{F}$ & 63 & White & $\mathrm{DTC}+\mathrm{MVC} *$ & $\sim 169$ & $<50$ & $<12$ \\
\hline WWH-B008 & M & 64 & Black & Descovy/Truvada & $\sim 47$ & $<50$ & $\sim 60$ \\
\hline WWH-B011 & M & 55 & White & Odesfy & $\sim 76$ & $<50$ & $\sim 264$ \\
\hline WWH-B012 & $\mathrm{F}$ & 52 & White & Odesfy & $\sim 98$ & $<50$ & $<12$ \\
\hline
\end{tabular}


Table 2. ART-naive participant clinical data

\begin{tabular}{|c|c|c|c|c|c|c|c|}
\hline Participant ID & Sex & Age (yr) & $\begin{array}{c}\text { Viral load (copies/ } \\
\mathrm{mL} \text { ) }\end{array}$ & CD4 counts cells $/ \mathrm{mm}^{3}$ & HIV dx year & ART status & Visit 1 (year) \\
\hline B465 & $M$ & 47 & 61,830 & 352 & 2012 & Naive & 2017 \\
\hline B461 & M & 49 & 42,620 & 333 & 2012 & Naive & 2017 \\
\hline 0M5374 & M & 29 & 96,125 & 214 & 2018 & Naive & 2018 \\
\hline
\end{tabular}

ure 7). In HIVE assays in which we were unable to recover more than 3 million viable cells, all cells were plated in QVOAs to maximize the accuracy of our IUPM calculations without a matched measurement for HIV DNA. Summary data for HIV DNA showed no significant decreases following treatment with anti-CD3/antiCD28 alone or with anti-CD3/anti-CD28 + ABT-199 $(P=0.16$ and $P=0.23$, respectively; Figure $8 \mathrm{~A})$. Treatment with anti-CD3/antiCD28 + HIV-specific effectors led to overall significant decreases in HIV DNA $(P=0.02)$, which were also observed with the addition of ABT-199 ( $P=0.03$, Figure $8 \mathrm{~A})$. In terms of intact-inducible reservoirs, QVOA results showed no significant decreases in IUPM when comparing the no-treatment condition to either anti-CD3/anti-CD28, anti-CD3/anti-CD28 + ABT-199, or antiCD3/anti-CD28 + HIV-specific effectors (Figure 8B). However, when cells were treated with the triple combination of anti-CD3/ anti-CD28, HIV-specific effectors, and ABT-199, we observed significant decreases in IUPM compared with the no-treatment condition ( $P=0.03$; Figure $8 \mathrm{~B})$. When considered individually, as in Figure 7, D and E, decreases in QVOA were also significant for 9/10 HIVE assays following treatment with the triple combination. Differences in IUPM were also significant when directly comparing anti-CD3/anti-CD28 + ABT-199 to the triple combination of anti-CD3/anti-CD28 + HIV-specific effectors + ABT-199 $(P=$ 0.02 , Figure $8 \mathrm{D})$. In conclusion, while cells harboring intact-inducible HIV reservoirs - as measured by QVOA - were not reduced following treatment with anti-CD3/anti-CD28 and either ABT-199 or HIV-specific T cell effectors, the combination of all 3 treatments unlocked consistent reductions in viral reservoirs in ex vivo $\mathrm{CD} 4^{+} \mathrm{T}$ cells from ART-suppressed individuals.

\section{Discussion}

The interaction with a CTL that results in target cell elimination is a highly regulated process on the part of both cells. In the context of HIV, the factors that define an effective CTL have been the subject of considerable study, which has led to numerous key insights, such as the role of $\mathrm{T}$ cell exhaustion in limiting immune control of viral replication $(7,52-56)$. In contrast, little is known about how intrinsic differences in infected-cell sensitivity may influence the outcome of interactions with CTL. The potential importance of this consideration is highlighted by oncology studies in which intrinsic resistance to CTL on the part of target cells has emerged as a key factor limiting the efficacy of immunotherapies $(57,58)$. In a recent study, we highlighted the potential role of intrinsic target cell resistance to CTL killing in HIV persistence. In the current study, we provide evidence for a causal role for BCL-2 in this resistance by demonstrating that a selective BCL-2 antagonist, ABT-199, enabled reductions in ex vivo infectious reservoirs following latency reversal and coculture with HIV-specific T cells. The transcriptional profiling performed here has also identified additional candidate mechanisms of resistance that will be pursued in future studies.

Our data with ex vivo CD4 ${ }^{+} \mathrm{T}$ cells indicate that ABT-199 acted by enabling CTL killing, since significant reductions were not observed with either LRA + CTL or with LRA + ABT-199. However, this must be contextualized within a more complex landscape, given that BCL-2 antagonism can also drive apoptosis through CTL-independent mechanisms (59-61). Overall, we interpret the results of our study to indicate that ABT-199 contributed to cell death by 3 mechanisms, depending on the source and infection status of the target cells. First, we consistently observed appreciable losses in the overall viability of both ex vivo and latency model cells, independent of any CTL recognition - likely as a result of an overall skewing of the anti-/proapoptotic balance maintained by BCL-2. This nonspecific induction of apoptosis was substantially mitigated by bryostatin-1, which is known to suppress apoptosis by phosphorylating and thus activating BCL-2 (62), and to a lesser degree by anti-CD3/anti-CD28 stimulation. We took care to control for any confounding effects arising from this nonspecific toxicity by calculating IUPM values based on only viable $\mathrm{CD} 4^{+} \mathrm{T}$ cells in each experimental condition. Second, in the TCM primary cell latency model, we observed dramatic reductions in relative frequencies of HIV-infected cells following treatment with ABT-199 (in the absence of CTL), indicating that HIV-infected cells were disproportionately susceptible to ABT-199-induced apoptosis compared with uninfected cells. This result can be explained by recent publications showing that Casp8p41 bound with BCL-2 in HIV-infected cells, which averted cell death (63). The BCL-2 antagonist ABT-199 can release Casp8p41 and specifically enhance the susceptibility of HIV-infected cells to viral cytopathicity (64). Interestingly, and in contrast to results from this latency model, viral cytopathicity did not appear to be sufficient to drive the death of reservoir-harboring cells from ex vivo CD4 ${ }^{+}$T cells, even in the presence of ABT-199. Rather, ABT-199 facilitated the death of ex vivo, HIV-infected cells by a third, CTL-dependent mechanism, consistent with the central hypothesis of the current study.

The mechanisms underlying our observation that infected cells in a primary cell latency model were susceptible to elimination by ABT-199 + LRA, whereas those in ex vivo CD4 ${ }^{+} \mathrm{T}$ cells were not, are currently unknown. However, these results appear to mirror our prior inability to reduce infectious reservoirs with combinations of CTL and LRAs, whereas such combinations are effective against models of latency $(13,21,65)$. We suggest that these results fit an emerging pattern in the field, as also evident in the lack of reservoir reductions in clinical trials, supporting the 
A

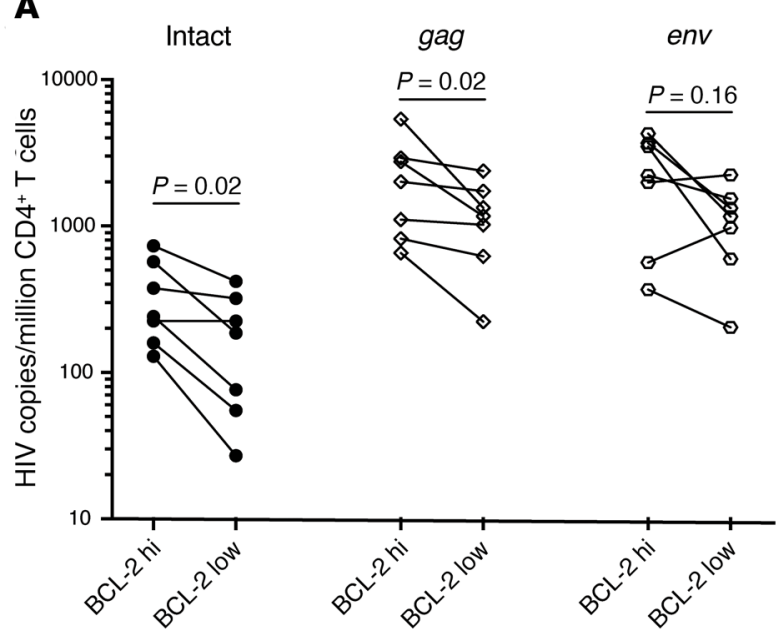

B

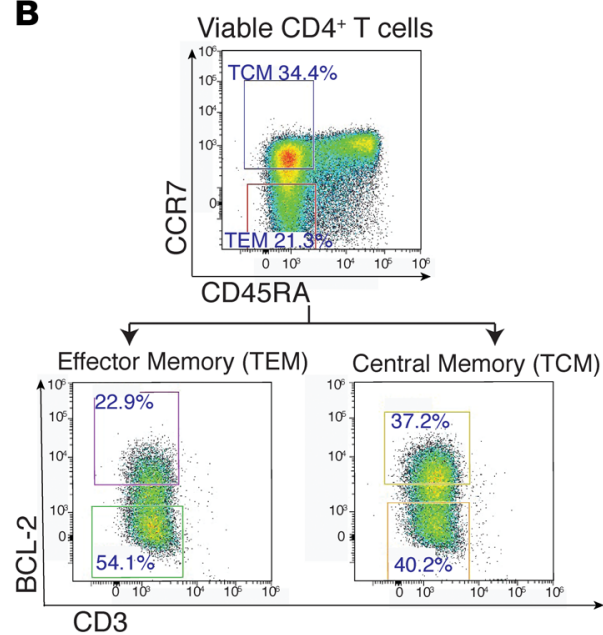

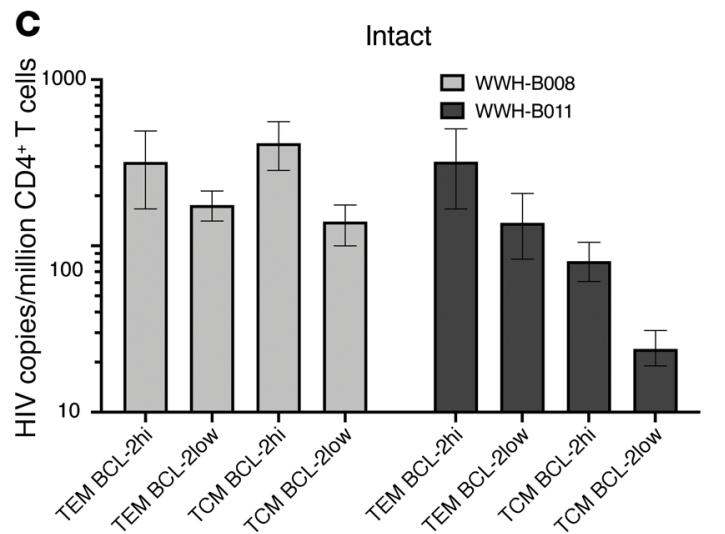

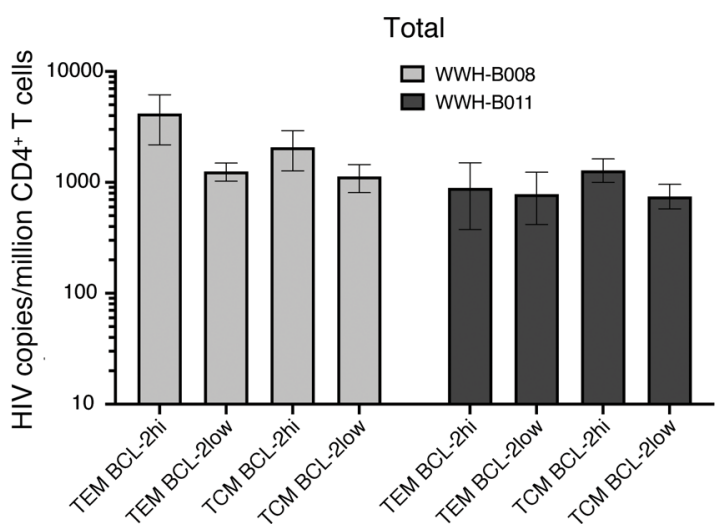

Figure 4. Intact HIV proviruses are preferentially harbored in BCL-2 $2^{\text {hi }}$-expressing CD4+ $\mathbf{T}$ cells ex vivo. (A) Shown are ddPCR results quantifying HIV DNA in resting ex vivo $C D 4^{+} T$ cells from ARV-treated donors that had been flow cytometry sorted based on BCL-2 expression. Intact quantification based on droplets that were double-positive for gag and env signals (represent full-length proviruses); gag, quantification based on any droplet amplified with gag primer/probes; env, quantification based on any droplet that amplified with env primer/probes (Wilcoxon's matched-pairs signed-rank test, $n=7$ ). (B) Flow cytometry plots depicting sorting based on both memory phenotype and BCL-2 expression, using CD45RA and CCR7 to separate TCM and TEM populations. (C) Intact and gag (see A) ddPCR results on samples from 2 ARV-treated donors, WWH-B008 (corresponds to flow plots in B), and WWH-B011. Note that the difference in presentation and analysis of these ddPCR data versus other ddPCR data in the manuscript is due to the low DNA yield after BCL-2 intracellular staining and flow sorting. Whereas in other experiments, each of 8 ddPCR replicates were treated as individual data points, here the ddPCR software (Quantasoft) generated maximum likelihood estimates of $95 \% \mathrm{Cl}$ (shown) based on the frequency of positive droplets for all 4 to 6 replicates taken together. This analysis method is recommended by the instrument manufacturer for the analysis of rare events.

idea that HIV reservoir-harboring cells - which durably persist in individuals on long-term ART - possess a resiliency that may not be reflected in short-term in vitro models of latency (22). The results of our study suggest one mechanism by which such resiliency could emerge, through infection of $\mathrm{CD} 4^{+} \mathrm{T}$ cells possessing natural variation in BCL-2 activity, followed by in vivo selection of an apoptosis-resistant BCL-2 $2^{\text {hi }}$ population. Initial support for this hypothesis is present in our observation of similar BCL-2 expression levels between $\mathrm{Gag}^{+}$and $\mathrm{Gag}^{-}$cells in ex vivo $\mathrm{CD}^{+} \mathrm{T}$ cells from 4 ART-naive individuals, in comparison with elevated BCL-2 expression in $\mathrm{Gag}^{+}$cells from individuals on long-term ART. In the ART-naive samples, the majority of ex vivo $\mathrm{Gag}^{+}$cells will have been newly infected with HIV and would have been destined to die shortly after this snapshot, with an average life span of approximately 2.2 days (66). In contrast, the infected cells reactivated from individuals on ART represent long-term survivors that were almost certainly infected before ART initiation (years prior). Recently, it has been demonstrated that the HIV reservoir is dynamically shaped by the proliferation and contraction of clones of HIV-infected cells (67), providing the replication and heritability needed to drive evolution within this surviving population, and the selection of prosurvival factors, such as BCL-2. A broader rendering of this hypothesis would include not only BCL-2, but other factors that influence the susceptibility of cells to apoptosis. One such factor is the anti-apoptotic protein BIRC5 (survivin), the expression of which was recently demonstrated to be overrepresented in clonally expanded cells in ex vivo $\mathrm{CD} 4^{+} \mathrm{T}$ cells (68). Such CTL-driven selection would be dependent upon the expression of antigen, and thus one may think that it would uniquely apply to cells harboring intact proviruses and not to those with defective proviruses. However, the dichotomy of intact proviruses being associated with antigen expression, while defective proviruses are 
A
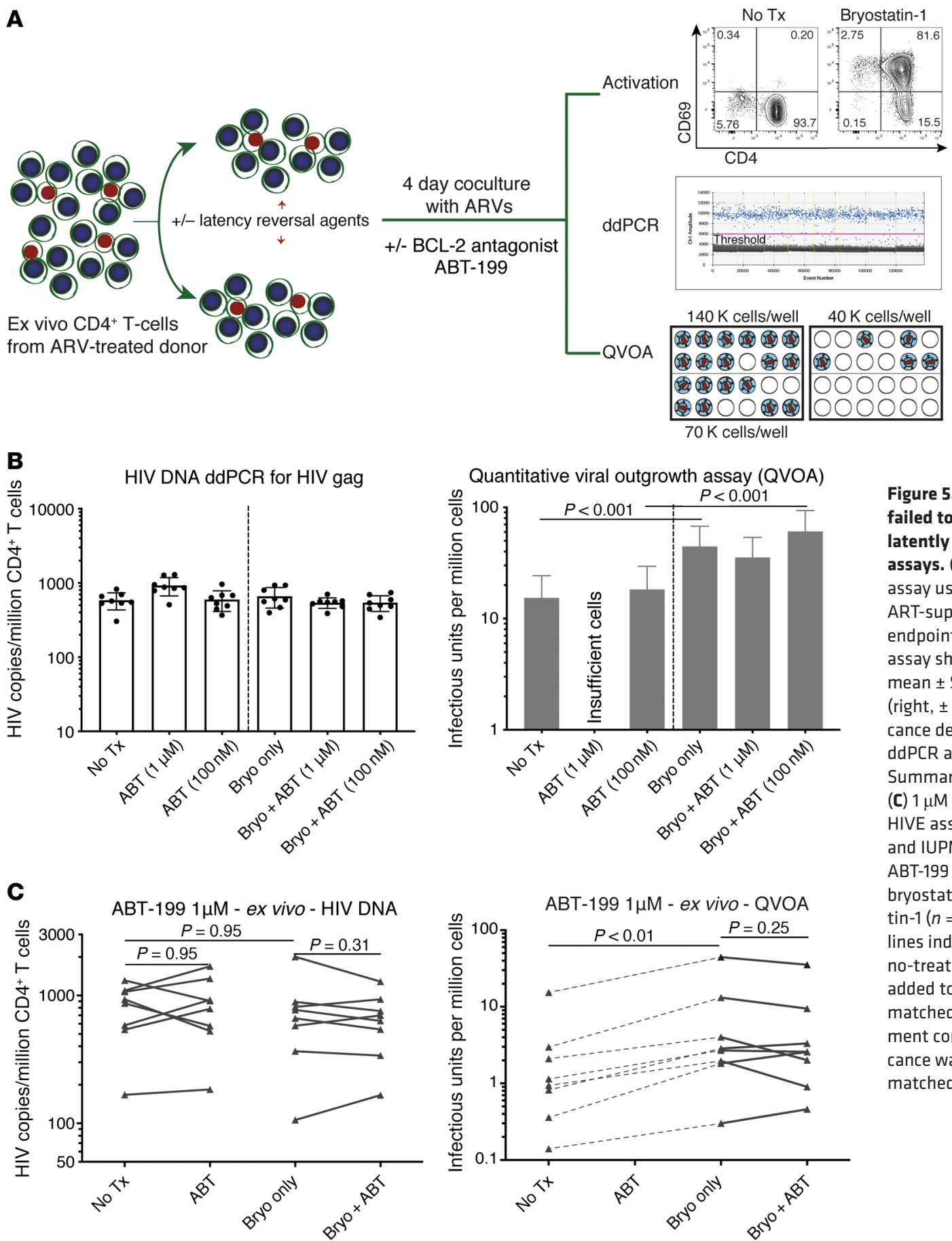

Figure 5. BCL-2 antagonist ABT-199

failed to drive reductions in ex vivo, latently infected $\mathrm{CD4}^{+} \mathrm{T}$ cells in HIVE assays. (A) Schematic of a HIVE assay using ex vivo $C D 4^{+} T$ cells from ART-suppressed individual showing endpoints. (B) A representative HIVE assay showing total HIV DNA (left, mean \pm SD of 8 replicates) and IUPM (right, $\pm 95 \% \mathrm{Cl}$ ). Statistical significance determined by 1-way ANOVA for ddPCR and a pairwise $\chi^{2}$ test for QVOA. Summary data for ABT-199 tested at (C) $1 \mu \mathrm{M}$ and (D) $100 \mathrm{nM}$ in following HIVE assays. Levels of HIV DNA (left) and IUPM (right) are shown, comparing ABT-199 alone versus no treatment and bryostatin-1 + ABT-199 versus bryostatin-1 $(n=8$ for $\mathbf{C}, n=6$ for $\mathbf{D})$. Dashed lines indicate paired bryostain-1 versus no-treatment conditions. DMSO was added to no-treatment conditions at a matched concentration with + treatment conditions. Statistical significance was determined by Wilcoxon's matched-pairs signed-rank test.
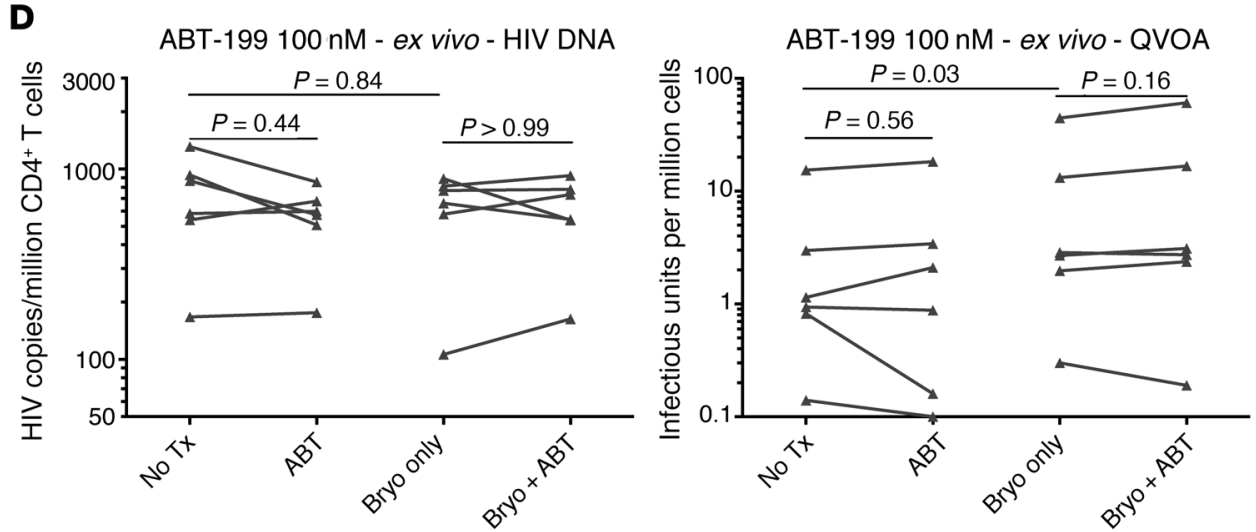
$\mathbf{A}$
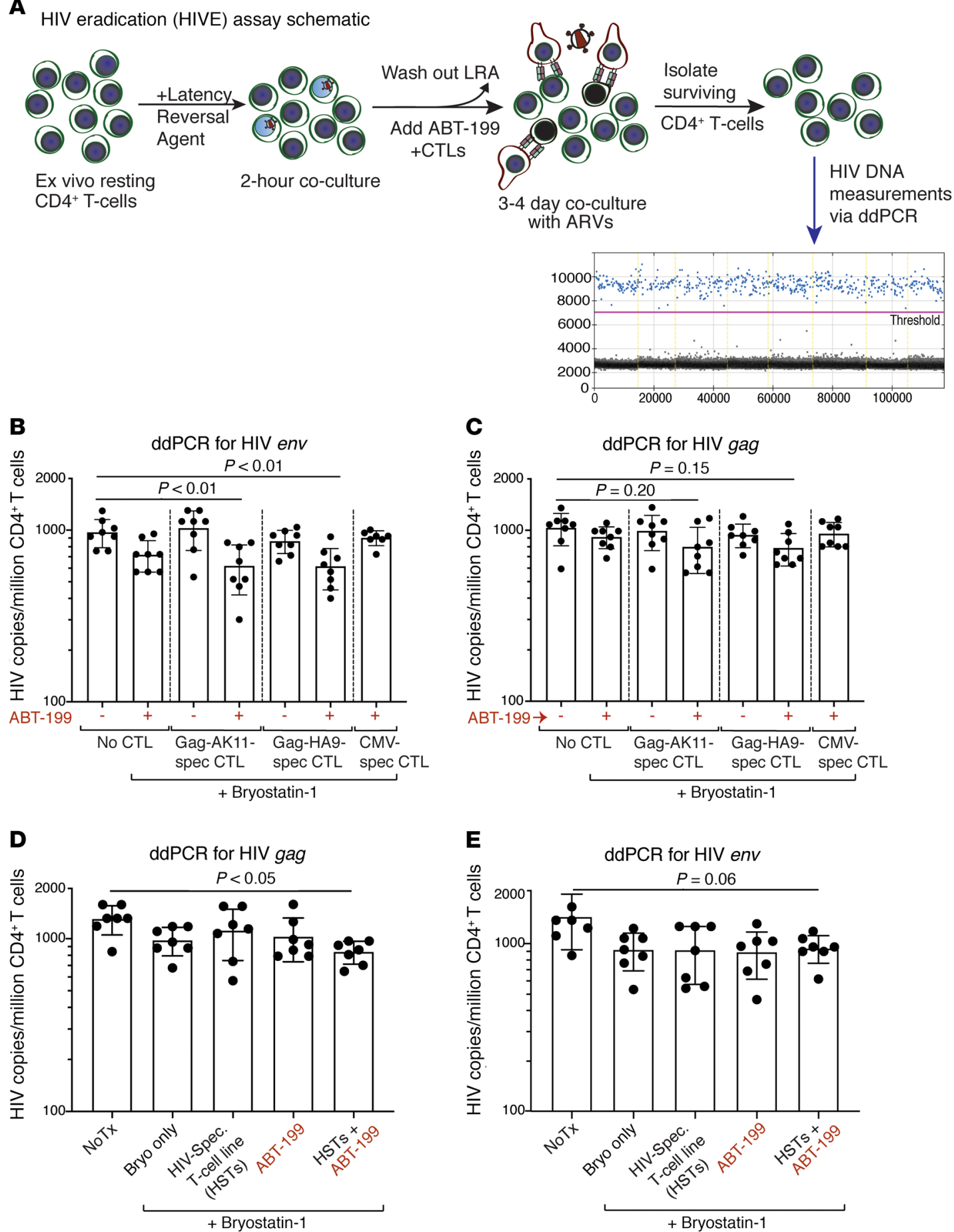

Figure 6. ABT-199 enables modest reductions in HIV-infected cells by HIV-specific T cell effectors, following reactivation with bryostatin-1. (A) Schematic of the HIVE assay with ddPCR as the endpoint. (B-E) ddPCR data measuring HIV-env (B and E) or HIV-gag (C and D) in DNA from HIVE assay samples, as indicated. Shown are mean \pm SD values of 8 replicates per sample (following exclusion of outliers based on a prespecified criterion; see Methods). $P$ values were calculated by ordinary 1-way ANOVA, with Tukey's multiple comparisons test if ANOVA test was significant.

not, does not fully reflect the complexity of the proviral landscape. A subset of defective proviruses are capable of expressing antigen and thus of being recognized by CTL $(69,70)$. On the other hand, a large proportion of intact proviruses are not expressed, even following stimulation with maximal LRAs, and thus are unlikely to express antigen in vivo. We suggest that this complexity underlies our observations that, whereas for some individuals only "intact" proviruses were enriched in BCL-2 ${ }^{\text {hi }}$ cells (e.g., WWH-B011 in Figure $4 \mathrm{C}$ ), in other individuals, a degree of enrichment was also observed for total HIV DNA (mostly defective) (Figure 4A). We also note that, whereas BCL-2 antagonism was required to achieve measurable reductions in QVOA assays, it also further enhanced 


\section{A}

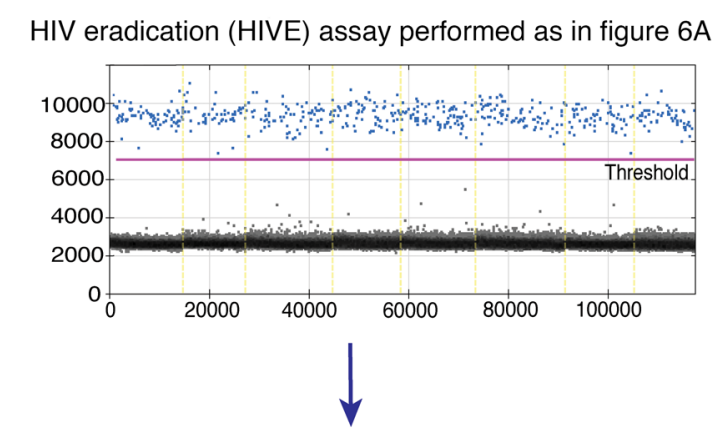

B

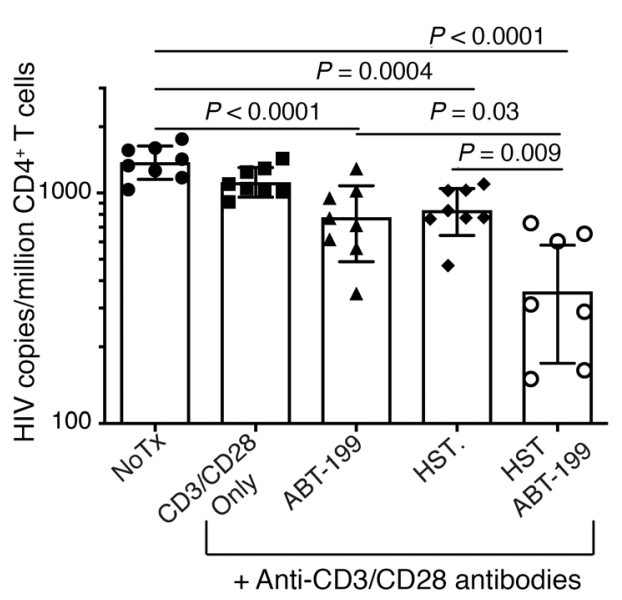

C

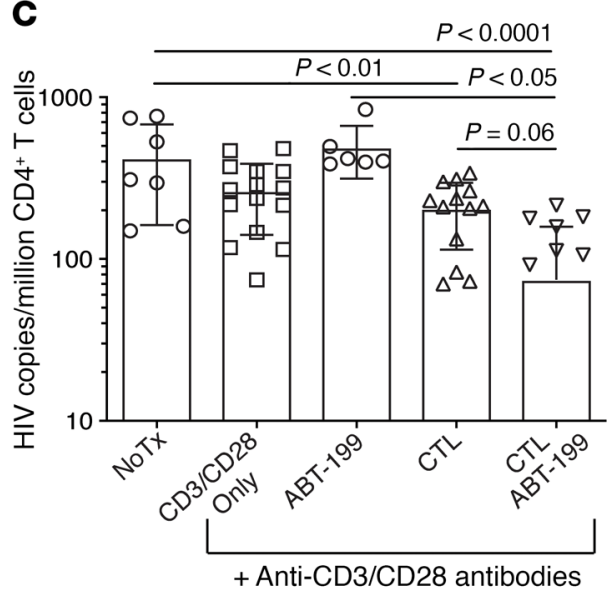

D

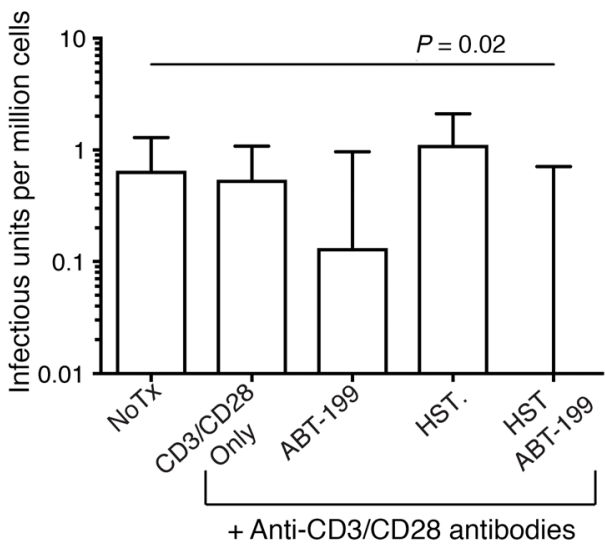

E

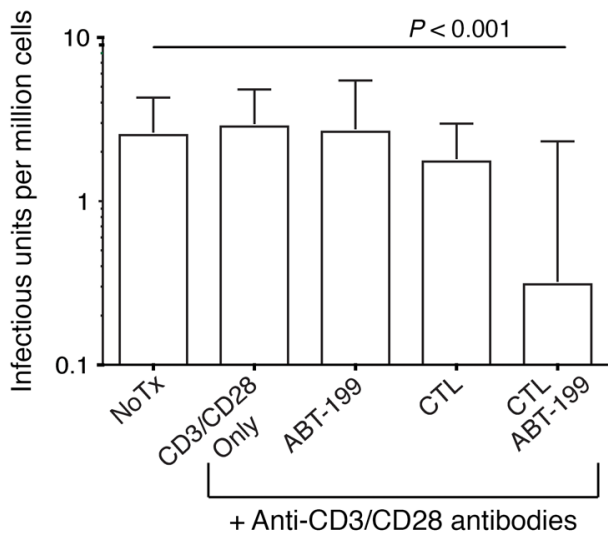

Obtain viable cell count via flow cytometry and use to aliquot cells for ...
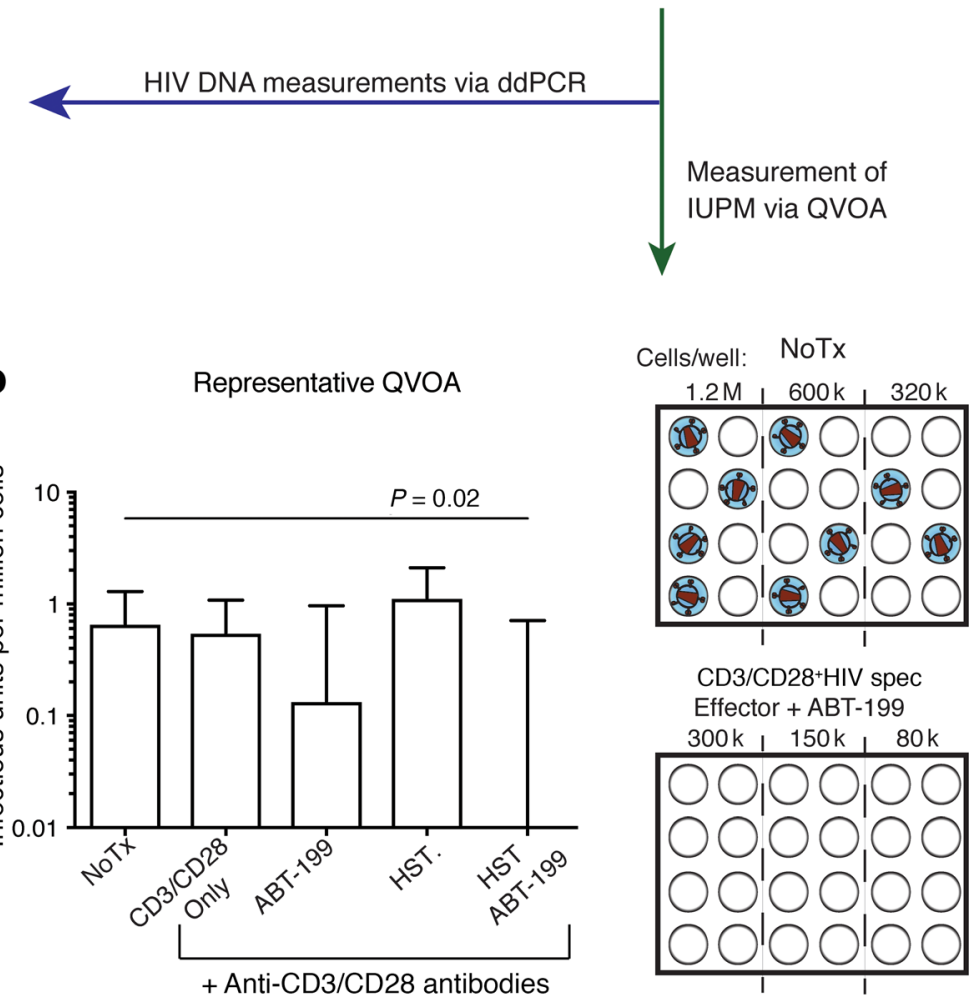

Cells/well: NoTx
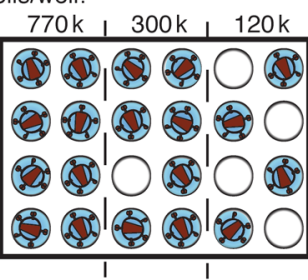

CD3/CD28+HIV spec

Effector + ABT-199 $260 \mathrm{k}+100 \mathrm{k}$ - 40k

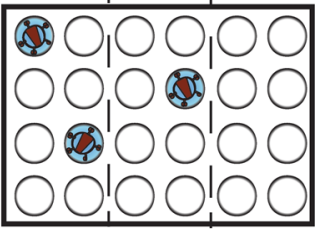

Figure 7. ABT-199 enables CTL-mediated reductions in ex vivo HIV reservoirs following reactivation with anti-CD3/anti-CD28. (A) Schematic of the HIVE assay showing representative endpoints. (B and C) Representative ddPCR data (mean \pm SD of 8-12 replicates) from 2 HIVE assays using autologous HSTs (B) and an autologous HIV-specific CTL clone (C). $P$ values were determined by 1-way ANOVA. (D and E) Representative QVOA data showing maximum likelihood estimates of IUPM $\pm 95 \% \mathrm{Cl}$ (the same HIVE assays in B and $\mathbf{C}$ ). $P$ values were determined by pairwise $\chi^{2}$ test. The representative QVOA plates shown on the right correspond to the no-treatment and the anti-CD3/anti-CD28 + HIV-specific effector + ABT-199 conditions.

CTL-mediated reductions in total HIV DNA (Figure 7, B and C), further supporting the idea that a subset of defective proviruses are harbored from CTL in BCL-2 ${ }^{\text {hi }}$ cells. Additional longitudinal studies of the expression of BCL-2 and other survival factors in ex vivo reservoir-harboring cells are needed to further test the hypothesis that CTLs select for infected cells with these prosurvival phenotypes. Pairing such studies with profiling of proviral landscapes and integration sites would allow further scrutiny of this hypothesis by assessing whether such selection is limited to cells harboring proviruses that are likely to drive antigen expression.

The results of our study suggest the possibility of adding BCL-2 antagonist to therapeutic combinations of CTLs and 


\section{A}

No Tx vs. treatments - total HIV DNA comparisons
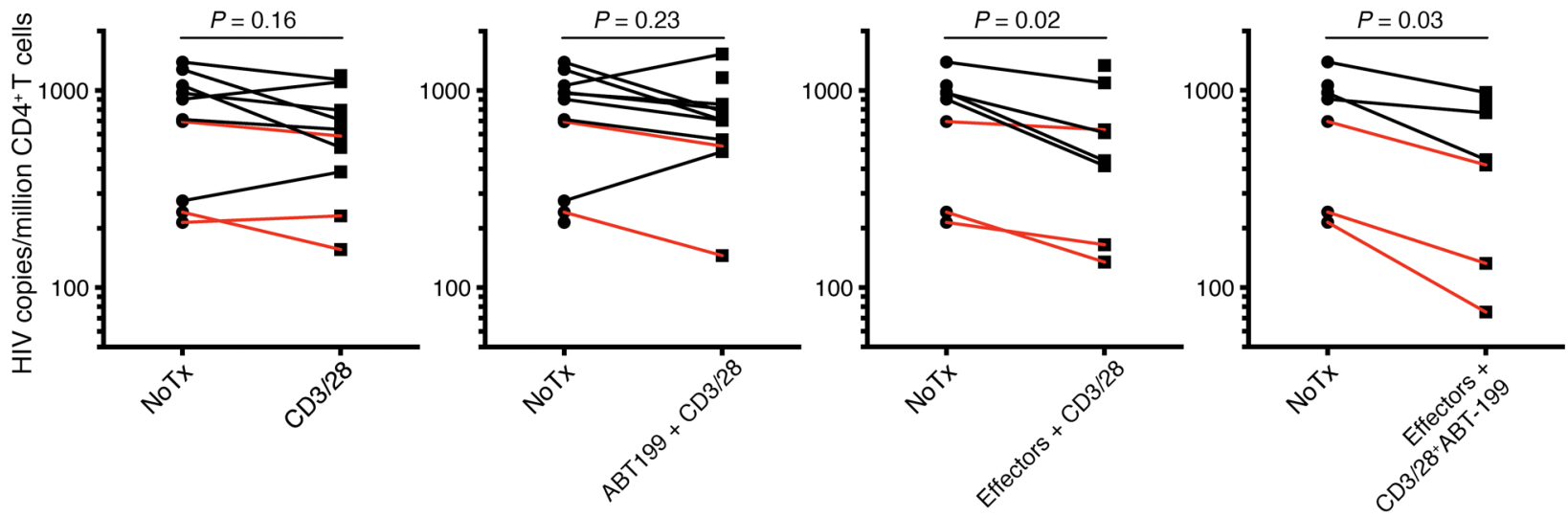

B

No Tx vs. treatments - QVOA IUPM comparisons
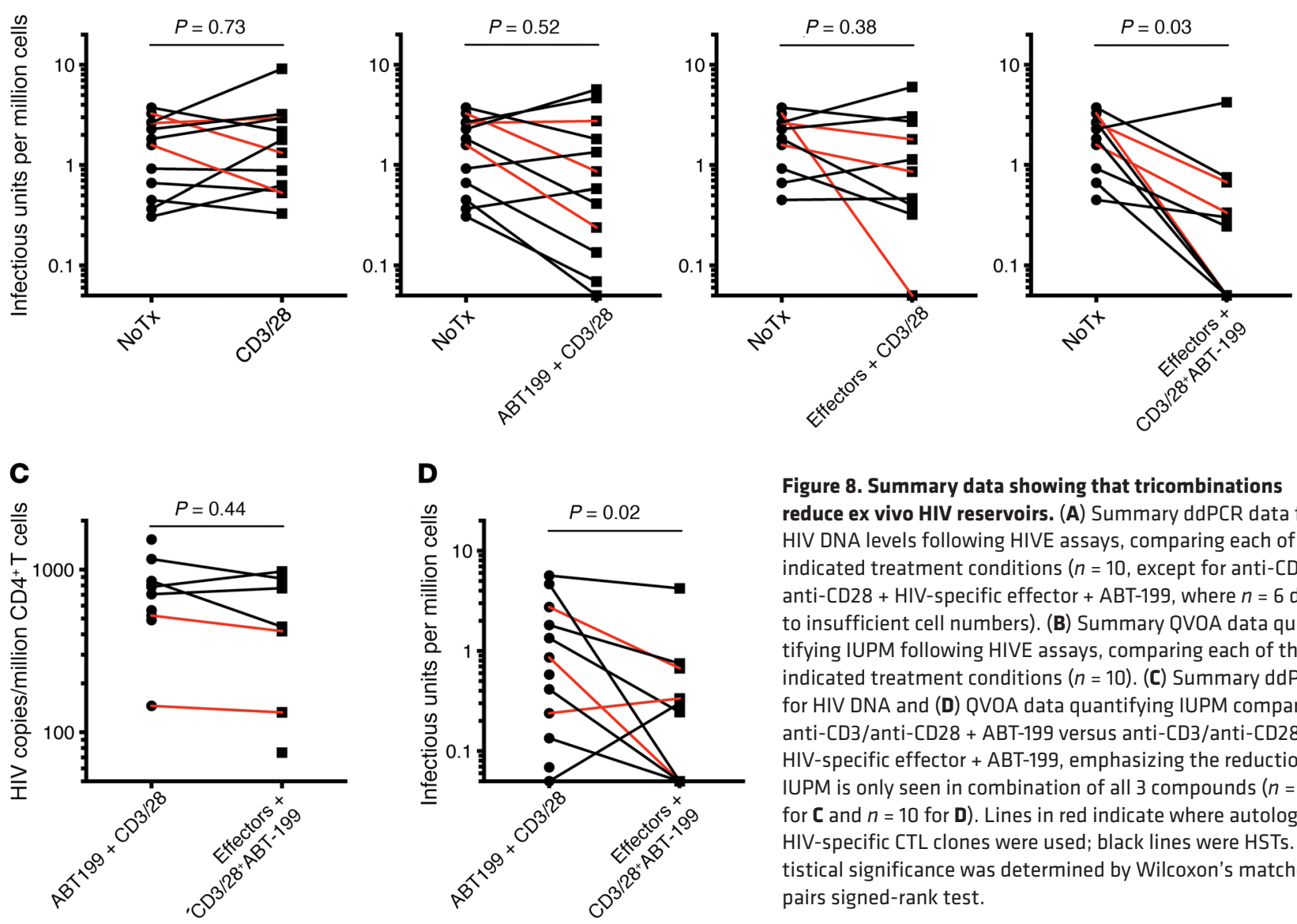

Figure 8. Summary data showing that tricombinations reduce ex vivo HIV reservoirs. (A) Summary ddPCR data for HIV DNA levels following HIVE assays, comparing each of the indicated treatment conditions ( $n=10$, except for anti-CD3/ anti-CD28 + HIV-specific effector + ABT-199, where $n=6$ due to insufficient cell numbers). (B) Summary QVOA data quantifying IUPM following HIVE assays, comparing each of the indicated treatment conditions $(n=10)$. (C) Summary ddPCR for HIV DNA and (D) QVOA data quantifying IUPM comparing anti-CD3/anti-CD28 + ABT-199 versus anti-CD3/anti-CD28 + HIV-specific effector + ABT-199, emphasizing the reduction of IUPM is only seen in combination of all 3 compounds $(n=6$ for $\mathbf{C}$ and $n=10$ for $\mathbf{D}$ ). Lines in red indicate where autologous HIV-specific CTL clones were used; black lines were HSTs. Statistical significance was determined by Wilcoxon's matchedpairs signed-rank test.

LRAs with the aim of achieving the in vivo reductions in HIV reservoirs that have eluded clinical trials to date. The BCL-2 antagonist ABT-199 used in the current study is the active ingredient in the licensed drug Venclexta (venetoclax), which is used to treat chronic lymphocytic leukemia (CLL) (71). Although venetoclax has nontrivial side effects, it is reasonably well tolerated, with CLL patients often taking this drug for years (72). It is therefore worth considering clinical trials involving the BCL-2 antagonist as a possible strategy for reducing or eliminating HIV reservoirs in individuals on long-term ART. As our ex vivo results suggest that the coordination of these agents with HIV-specific CTL may be needed to achieve such reductions, it is important to note that ABT-199 did not impair the viability or functionality of $\mathrm{CD}^{+} \mathrm{T}$ cells (see also ref. 73). Moreover, in a murine cancer model, venetoclax enhanced anti-PD-1-mediated $\mathrm{T}$ cell antitumor activity $(73,74)$. In conclusion, the current study provides evidence that HIV reservoir-harboring cells have been selected for survivability, conferred, at least in part, through BCL-2. This establishes a ratio- 
nale for the development of novel tripartite therapies incorporating LRA, BCL-2 antagonism, and enhancement of $\mathrm{CD}^{+} \mathrm{T}$ cell responses through immunotherapy, cell therapy, or vaccination to reduce or eliminate HIV reservoirs.

\section{Methods}

LRA, chemical agents, and antibodies. LRAs and BCL-2 antagonist were used at the following concentrations: bryostatin-1 dissolved in DMSO and used at $10 \mathrm{nM}$ (Sigma-Aldrich); anti-CD3 (clone OKT3, BioLegend), anti-CD28 (clone CD28.2, BioLegend), and anti-CD3/ anti-CD28 antibodies were used at $1 \mu \mathrm{g} / \mathrm{mL}$ each; PMA and ionomycin were dissolved in DMSO, and PMA was used at $25 \mathrm{nM}$ (SigmaAldrich), ionomycin at $1 \mu \mathrm{g} / \mathrm{mL}$ (Sigma-Aldrich); ABT-199 (Med Chem Express, catalog HY-15531) was dissolved in DMSO used at $1 \mu \mathrm{M}$ or $100 \mathrm{nM}$ (as indicated). Fixable viability dye (aqua, Thermo Fisher Scientific), anti-human CD3 (clone SK7, BD Biosciences), antihuman CD4 (clone RPA-T4, BD Biosciences), anti-human CD8 (clone RPA-T8, BioLegend), anti-human CD45RA (clone HI100, BD Biosciences), anti-human CCR7 (clone G043H7, BioLegend), anti-human CD69 (clone FN50, BioLegend), anti-human HLA-DR (clone L243, BioLegend), anti-human BCL-2 (clone 100, BioLegend), and p24 antibodies (anti-HIV core antigen: clone KC57, Beckman Coulter; p24.2 clone 28B7, MediMabs) were used.

Peptide-pulse CTL killing assay. CD4 ${ }^{+} \mathrm{T}$ cells were enriched from PBMCs by magnetic negative selection, following the manufacturer's instructions (STEMCELL Technologies). Where indicated, these cells were activated before peptide pulsing with $1 \mu \mathrm{g} / \mathrm{mL}$ each anti-CD3 and anti-CD28 antibodies in RPMI-10 media supplemented with 50 $\mathrm{U} / \mathrm{mL}$ of IL-2 (R10-50). Purified $\mathrm{CD}^{+} \mathrm{T}$ cells were then pulsed with RR11 peptide (RLRDLLLIVTR) (Genscript) at the indicated concentrations for 30 minutes in R10-50. CD4 ${ }^{+} \mathrm{T}$ cells were then washed and cocultured with autologous, RR11-specific CTL clones in R10-50. After 16 hours, cell cultures were stained with anti-human CD3, CD4, and CD8 antibodies and viability dye. In some experiments, cells were also stained with anti-human CD45RA and CCR7 antibodies. Cells were then treated with Fixation/Permeabilization Solution (BD Biosciences), followed with BCL-2 intracellular staining. Samples were analyzed by flow cytometry, and data analysis was performed with FlowJo, version 10, software.

RNA-Seq sample acquisition. Cultured TCM CD4+ $4^{+}$cells were generated as previously described (48) (see also Supplemental Methods). These TCM CD $4^{+} \mathrm{T}$ cells were divided into either a real or mock condition and then subdivided into 2 populations each, receiving either CFSE or CTFR labeling (Thermo Fisher Scientific). After staining, $\mathrm{CFSE}^{+}$cells in the real condition were pulsed with $1 \mu \mathrm{g} / \mathrm{mL}$ of RR11 peptide for 30 minutes. Following extensive washing, peptide-pulsed cells were mixed with equal numbers of unpulsed $\mathrm{CTFR}^{+}$cells and cocultured with CTL clones at an effector/target ratio of 1:1 overnight in R10-50 media. Cells from the mock condition did not receive peptide, but were otherwise treated identically to those of the real condition. Following the overnight culture, cells were stained with antibodies against human CD3, CD4, and CD8 and DAPI and then sorted by FACS Influx (BD Biosciences) directly into vessels containing lysis buffer (QIAGEN Inc.). Total RNA was immediately extracted using the RNeasy Micro Kit (QIAGEN Inc.), and RNA quality and concentration were determined by Agilent Bioanalyzer 2100. Library preparation used the methods of TruSeq RNA Sample Preparation (nonstranded and poly-A selection), and sequencing was run on HiSeq 4000 (Illumina) with a single read clustering and 50 cycles of sequencing.

RNA-Seq data analysis. The raw sequencing reads in BCL format were processed through bcl2fastq 2.19 (Illumina) for FASTQ conversion and demultiplexing. RNA reads were aligned and mapped to the GRCh37 human reference genome by STAR (version 2.5.2) (https:// github.com/alexdobin/STAR) (75), and transcriptome reconstruction was performed by Cufflinks (version 2.1.1) (http://cole-trapnell-lab. github.io/cufflinks). The abundance of transcripts was measured with Cufflinks in FPKM $(76,77)$. Gene expression profiles were constructed for differential expression, cluster, and principle component analyses with the DESeq2 package (https://bioconductor.org/packages/ release/bioc/html/DESeq2.html) (78). For differential expression analysis, pairwise comparisons between 2 or more groups using parametric tests where read counts followed a negative binomial distribution with a gene-specific dispersion parameter were used. Corrected $P$ values were calculated based on the Benjamini-Hochberg method to adjusted for multiple testing. The list of differentially expressed genes comparing "real survivors" and "real bystanders" was determined with a cutoff of FDR (adjusted $P$ value) of less than 0.05. Pathway analysis was performed with IPA (QIAGEN Inc.) (79). Markedly enriched pathways were selected with a threshold of Benjamini-Hochberg multiple testing correction of $P<0.05$ and displayed as $-\log ($ Benjamini-Hochberg, $P$ value) of more than 1.3. Molecular networks were also analyzed using IPA, with the top 25 significantly enriched networks shown in Supplemental Table 1. RNA-Seq data were deposited in the NCBI's Gene Expression Omnibus database (GEO GSE143879).

HIV flow direct staining of HIV-infected cells in ex vivo $C D 4^{+} T$ cells. Staining of HIV-infected cells was performed as previously described (44). Briefly, CD4 ${ }^{+} \mathrm{T}$ cells were activated with PMA $(25 \mathrm{nM})$ and ionomycin $(1 \mu \mathrm{g} / \mathrm{mL})$ for 24 hours at $37^{\circ} \mathrm{C}$ in $\mathrm{R}-10$ media and then harvested for flow cytometry. For each sample, around 4-8 $\times 10^{6}$ cells were stained with viability dye; anti-human CD3, CD4, and CD8; and 2 intracellular antibodies targeting HIV core antigen and anti-human BCL-2 and then analyzed by flow cytometry (Attune NxT, Thermo Fisher Scientific).

Sorting for $B C L-2^{\text {hi }}$ versus $B C L-2^{\text {lo }}$ cells. From 5 to $10 \times 10^{6}$ resting $\mathrm{CD}^{+}{ }^{+} \mathrm{T}$ cells from long-term ARV-treated participants' PBMCs were enriched by negative selection. Cells were used for flow cytometry staining with surface antibodies for 30 minutes $4^{\circ} \mathrm{C}$ using viability dye and antibodies anti-human CD3, CD4, and CD8 with (Figure 4, B and C) or without (Figure 4A) CD45RA and CCR7. After washing out the extra surface antibodies, cells were fixed with BioLegend Fixation Buffer for 5 minutes in $4^{\circ} \mathrm{C}$ and then permeabilized with BioLegend Permeabilization Wash Buffer and stained intracellularly with anti-human BCL-2 for 30 minutes. Finally, cells were resuspended in MACS buffer and analyzed/sorted by flow cytometry (SONY MA9000) based on BCL-2 high versus low. Sorted cells were pelleted, and DNA was extracted with the QIAamp DNA FFPE Tissue Kit (QIAGEN Inc.) following the manufacturer's protocol. DNA was used for intact proviral DNA assays (IPDAs).

HIVE assays. HIVE assays were set up as previously described (21). Briefly, more than $20 \times 10^{6}$ resting $\mathrm{CD}^{+} \mathrm{T}$ cells were pulsed with bryostatin-1 or anti-CD3/anti-CD28 antibodies for 2 hours, then washed and cocultured with or without ABT-199 and/or HIV-specific effectors (as indicated in the figures) in HIVE media: XVIVO-15 medium (Lonza) supplemented with $1 \mu \mathrm{M}$ tenofovir disoproxil fumarate, $1 \mu \mathrm{M}$ 
nevirapine, $1 \mu \mathrm{M}$ emtricitabine, $10 \mu \mathrm{M}$ T-20, $10 \mathrm{U} / \mathrm{mL}$ human DNAse I (ProSpec), and $0.1 \mathrm{nM} \mathrm{IL-7.} \mathrm{Following} \mathrm{a} \mathrm{3-} \mathrm{to} 4$-day coculture, CD4 ${ }^{+}$ $\mathrm{T}$ cells were isolated and rested for 24 hours in R10-50 media at $37^{\circ} \mathrm{C}$ to allow for ARV washout. Aliquots of pre- and post-CD4 enrichment samples were collected and stained for viability and memory phenotype/activation status with antibodies against anti-human CD3, CD4, CD8, CD45RA, CCR7, CD69, and HLA-DR, then analyzed by flow cytometry. Following the overnight culture, a small aliquot of cells was mixed with CountBright Absolute Counting Beads and viability dye (Invitrogen Technologies) to obtain a count of total, live CD4 ${ }^{+} \mathrm{T}$ cells by flow cytometry. This viable cell count was used to determine cell numbers for ddPCR and QVOA plating strategies.

ddPCR. ddPCR measuring total HIV DNA (HIVEs) was performed as previously described (80), with slight modifications. For each PCR reaction, 5 units of restriction enzyme BsaJI (NEB) was directly mixed with 300 ng of DNA, ddPCR Supermix (no dUTP) for probes (Bio-Rad), and final concentrations of $900 \mathrm{nM}$ for the primers and $250 \mathrm{nM}$ for the probe. Primers and probes were as follows: RPP30 forward primer, GATTTGGACCTGCGAGCG; reverse primer, GCGGCTGTCTCCACAAGT; probe, VIC-CTGAACTGAAGGCTCT-MGBNFQ; HIV-gag forward primer, TCTCGACGCAGGACTCG; reverse primer, TACTGACGCTCTCGCACC; and probe, FAM-CTCTCTCCTTCTAGCCTC-MGBNFQ. Droplets were prepared using the QX200 Droplet Generator (Bio-Rad) following the manufacturer's instructions. Sealed plates were cycled using the following program: $95^{\circ} \mathrm{C}$ for 10 minutes; 40 cycles of $94^{\circ} \mathrm{C}$ for $30 \mathrm{sec}-$ onds, $60^{\circ} \mathrm{C}$ for 1 minute; and $98^{\circ} \mathrm{C}$ for 10 minutes. Reactions were analyzed using the QX200 Droplet Reader and number of template molecules/ $\mu \mathrm{L}$ of starting material was estimated using the Quantalife ddPCR software. Eight technical replicates were run per sample, and we consistently applied a predetermined exclusion criterion to outliers that deviated from mean values by more than 2 times the SD.

For BCL-2 sorted samples (Figure 4) and HIVEs shown in Figure 6, a modified IPDA (45) was applied. For each PCR reaction, the same ddPCR supermix and final concentrations of primers and probes as above were used, but with 5 units of restriction enzyme Xho I (NEB) mixed with 750 ng DNA (HIVEs) or approximately 250 ng DNA (BCL-2 sorted samples, low DNA yield after intracellular staining and flow sorting). Primers and probes were used in 2 separate PCR systems: the first system was housekeeping multiplex with RPP30 (same as above) and RPP30-shearing forward primer, CCATTTGCTGCTCCTTGGG; reverse primer, CATGCAAAGGAGGAAGCCG; probe, FAM-GGAAAGGAGCAAGGTTC-IABkFQ. The second system was HIV multiplex with gag primers/probe

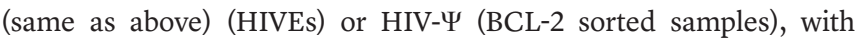
forward primer, CAGGACTCGGCTTGCTGAAG; reverse primer, GCACCCATCTCTCTCCTTCTAGC; $\Psi$ probe, FAM-TTTTGGCGTACTCACCAGT-3IABKFQ; and HIV-env (RRE) forward primer, AGTGGTGCAGAGAGAAAAAAGAGC; reverse primer, GTCTGGCCTGTACCGTCAGC; env probe HEX-CCTTGGGTTCTTGGGA-IABkFQ; and hypermutant probe, IABkFQ-CCTTAGGTTCTTAGGAGC-IABkFQ (OM5011, OM5148, OM5267, WWH-B008, and WWH-B012). Because some samples (OM5334 and WWH-B011) showed low amplification efficiency on env signal, we designed alternative primers/probe to replace the $e n v$ primer/probe. The alternative primers/probe were as follows: Alt-env (RRE) forward primer, ACTATGGGCGCAGCGTC; reverse primer, CCCCAGACTGTGAGTTGCA;
Alt-env probe HEX-CTGGCCTGTACCGTCAG-3IABKFQ. The PCR program was as follows: $95^{\circ} \mathrm{C}$ for 10 minutes; 40 cycles of $94^{\circ} \mathrm{C}$ for 30 seconds, $53^{\circ} \mathrm{C}$ for 1 minute; and $98^{\circ} \mathrm{C}$ for 10 minutes. DNA input of housekeeping multiplex was 100-fold (HIVEs) or 30-fold (BCL-2 sorted samples) diluted from the input of the HIV-multiplex. Total gag, $e n v$, or intact provirus copies were calculated by multiplying the dilution factors, and intact provirus copies were corrected with the shearing percentage calculated from the housekeeping multiplex. For HIVEs, 8 technical replicates were run per sample. A predetermined exclusion criterion was applied to remove outliers that deviated from mean values by more than 2 times the SD. For BCL- 2 sorted samples, 4 to 6 technical replicates were run per sample, and merged data from QuantaSoft software were exported and analyzed.

QVOAs. QVOAs were performed using a previously described protocol (81), with slight modifications depending on the application. Live cells counted by flow cytometry were distributed into either 3 of 2 -fold serial dilutions with 8 or 12 replicates per dilution or 4 of 2 -fold serial dilutions with 24 replicates per dilution. This was determined based on the numbers of viable cells recovered at the end of each HIVE assay and the baseline IUPM values of the donor. At least $3 \times 10^{6}$ cells were plated for any given QVOA (where cell numbers fell below this threshold, QVOA assays were omitted). Cells were then stimulated with $2 \mu \mathrm{g} / \mathrm{mL}$ of PHA (Thermo Fisher Scientific) $+10^{6}$ PBMCs (HIV ${ }^{-}$donor, irradiated at $50 \mathrm{~Gy}$ ). The next day, $10^{6} \mathrm{CCR}^{+} \mathrm{MOLT}-4$ cells were added along with a half media change. Cultures were then incubated for 14 days, with half media changes with R10-50 every 3 to 4 days. We performed p24 ELISA on supernatant 15 days after the PHA stimulation. For each condition, values for cells/well, number of positive wells, and total wells tested were entered into a limiting dilution analyzer (http://bioinf.wehi.edu.au/software/elda/) to calculate the maximal likelihood IUPM and a corresponding 95\% CI.

Statistics. Statistical analyses were performed using Prism 7 (GraphPad), and the statistical analysis methods used are reported in the figure legends. Comparisons among different peptide concentrations used Student's $t$ test (2 tailed). Comparisons between BCL-2 MFI of $\mathrm{Gag}^{+}$versus Gag populations in HIV-flow used unpaired nonparametric test (2 tailed) Wilcoxon's signed-rank test. Comparisons between BCL- $2^{\text {hi }}$ versus BCL-2 ${ }^{\text {lo }}$ sorted samples used paired nonparametric test (2 tailed; Wilcoxon's matched-pairs signed-rank test). All ddPCR data of HIVEs were analyzed by ordinary 1-way ANOVA, with Tukey's multiple comparisons test if the ANOVA test was significant, and statistics for the summary data sets for HIV DNA were performed using the mean of 8 replicates per condition. QVOAs were run at the end of each HIVE assay, the IUPM was calculated as described above, and $\chi^{2}$ test was applied to determine the significance. All comparisons between HIVE conditions in the summary data used paired nonparametric test (2-tailed; Wilcoxon's matched-pairs signed-rank test). A $P$ value of less than 0.05 was considered significant.

Study approval. $\mathrm{HIV}^{+}$individuals were recruited from either the Maple Leaf Medical Clinic through a protocol approved by the University of Toronto (Toronto, Canada) IRB or Whitman-Walker Health in Washington, DC (Table 1). Some samples were also collected through an IRB-approved protocol at the Rockefeller University (New York, New York, USA) (Table 2). Additional use of deidentified samples was reviewed and approved by George Washington University and Weill Cornell Medicine IRBs. All subjects were adults and gave written informed consent before their participation. Leukapheresis samples 
were used immediately if possible or cryopreserved in liquid nitrogen; cells were not left in culture before the initiation of experiments.

\section{Author contributions}

RBJ, YR, and SHH conceptualized the study. RBJ, YR, SHH, SP, WDCA, DM, DA, ABM, AB, and CMB developed the methodology. RBJ, YR, SHH, SP, WDCA, DM, DA, ABM, RD, DC, EZ, TMM, RT, $\mathrm{TR}$, and CDM conducted the investigation. CMK, EB, AW, CC, and $\mathrm{WDH}$ provided resources in the form of clinical samples. RBJ, $\mathrm{YR}$, and SHH analyzed data and wrote the manuscript, which was reviewed by all authors. $\mathrm{RBJ}, \mathrm{AB}$, and $\mathrm{CMB}$ acquired funding. $\mathrm{RBJ}$ supervised the study. YR and SHH share first authorship. Authorship order for the 2 co-first authors was determined based on their time of entry into the project (YR entered the project before SHH).

\section{Acknowledgments}

We thank Marina Caskey for providing deidentified samples from ART-naive donors used in this study. We also thank Natalie Kinloch and Zabrina Brumme for designing the alternative ddPCR primers. We would like to thank the Genomics Resources Core Facility at Weill Cornell Medicine for performing sequenc- ing and data normalization. This work was supported by R01 grants AI31798 and AI147845 (NIH). It was also supported in part by the Martin Delaney BELIEVE Collaboratory (NIH grant 1UM1AI26617) and the NIH-funded Center for AIDS Research Grants (P30 AI117970), which are both supported by the following NIH Co-Funding and Participating Institutes and Centers: the National Institute of Allergy and Infectious Diseases (NIAID), the National Cancer Institute (NCI), the National Institute of Child Health and Human Development (NICHD), the National Heart, Lung, and Blood Institute (NHLBI), the National Institute on Drug Abuse (NIDA), the National Institute of Mental Health (NIMH), the National Institute on Aging (NIA), the Fogarty International Center (FIC), and the Office of AIDS Research (OAR). The following reagents were obtained from the NIH AIDS Research and Reference Reagent Program: IL-2, pNL4-3, and CCR5 ${ }^{+}$MOLT-4 cells. Reagents for HIV p24 ELISAs were obtained from the NCI's AIDS and Cancer Virus Program.

Address correspondence to: R. Brad Jones, 413 East 69th Street, BB-526, New York, New York 10021, USA. Phone: 646.962.2459; Email: rbjones@med.cornell.edu.
1. Turk G, et al. Early Gag immunodominance of the HIV-specific T-cell response during acute/ early infection is associated with higher CD8+ $\mathrm{T}$-cell antiviral activity and correlates with preservation of the CD4+ T-cell compartment. J Virol. 2013;87(13):7445-7462.

2. Turnbull EL, et al. HIV-1 epitope-specific CD8+ T cell responses strongly associated with delayed disease progression cross-recognize epitope variants efficiently. JImmunol. 2006;176(10):6130-6146.

3. Betts MR, et al. HIV nonprogressors preferentially maintain highly functional HIV-specific CD8+ T cells. Blood. 2006;107(12):4781-4789.

4. Altfeld M, et al. HLA alleles associated with delayed progression to AIDS contribute strongly to the initial CD8(+) T cell response against HIV-1. PLoS Med. 2006;3(10):e403.

5. Day CL, et al. Proliferative capacity of epitope-specific CD8 T-cell responses is inversely related to viral load in chronic human immunodeficiency virus type 1 infection. J Virol. 2007;81(1):434-438.

6. Cartwright EK, et al. CD8(+) lymphocytes are required for maintaining viral suppression in SIV-infected macaques treated with short-term antiretroviral therapy. Immunity. 2016;45(3):656-668.

7. Jones RB, Walker BD. HIV-specific CD8 ${ }^{+}$ $\mathrm{T}$ cells and HIV eradication. J Clin Invest. 2016;126(2):455-463.

8. Yang OO, et al. Suppression of human immunodeficiency virus type 1 replication by CD8+ cells: evidence for HLA class I-restricted triggering of cytolytic and noncytolytic mechanisms. J Virol. 1997;71(4):3120-3128.

9. Migueles SA, et al. Lytic granule loading of CD8+ $\mathrm{T}$ cells is required for HIV-infected cell elimination associated with immune control. Immunity. 2008;29(6):1009-1021.

10. Chun TW, Finzi D, Margolick J, Chadwick K, Schwartz D, Siliciano RF. In vivo fate of HIV-1- infected T cells: quantitative analysis of the transition to stable latency. Nat Med. 1995;1(12):1284-1290.

11. Finzi $\mathrm{D}$, et al. Identification of a reservoir for HIV-1 in patients on highly active antiretroviral therapy. Science. 1997;278(5341):1295-1300.

12. Wong JK, et al. Recovery of replication-competent HIV despite prolonged suppression of plasma viremia. Science. 1997;278(5341):1291-1295.

13. Shan L, et al. Stimulation of HIV-1-specific cytolytic T lymphocytes facilitates elimination of latent viral reservoir after virus reactivation. Immunity. 2012;36(3):491-501.

14. Deeks SG. HIV: Shock and kill. Nature. 2012;487(7408):439-440.

15. Søgaard OS, et al. The depsipeptide romidepsin reverses HIV-1 latency in vivo. PLoS Pathog. 2015;11(9):e1005142.

16. Rasmussen TA, et al. Activation of latent human immunodeficiency virus by the histone deacetylase inhibitor panobinostat: a pilot study to assess effects on the central nervous system. Open Forum Infect Dis. 2015;2(1):ofv037.

17. Rasmussen TA, et al. Panobinostat, a histone deacetylase inhibitor, for latent-virus reactivation in HIV-infected patients on suppressive antiretroviral therapy: a phase $1 / 2$, single group, clinical trial. Lancet HIV. 2014;1(1):e13-2.

18. Ke R, Lewin SR, Elliott JH, Perelson AS. Modeling the effects of vorinostat in vivo reveals both transient and delayed HIV transcriptional activation and minimal killing of latently infected cells. PLoS Pathog. 2015;11(10):e1005237.

19. van Praag RM, et al. OKT3 and IL-2 treatment for purging of the latent HIV-1 reservoir in vivo results in selective long-lasting CD4+ T cell depletion. J Clin Immunol. 2001;21(3):218-226.

20. Dybul M, et al. Pilot study of the effects of intermittent interleukin-2 on human immunodeficiency virus (HIV)-specific immune responses in patients treated during recently acquired HIV infection. J Infect Dis. 2002;185(1):61-68.

21. Huang SH, et al. Latent HIV reservoirs exhibit inherent resistance to elimination by CD8+ T cells. J Clin Invest. 2018;128(2):876-889.

22. Huang SH, McCann CD, Mota TM, Wang C, Lipkin SM, Jones RB. Have cells harboring the HIV reservoir been immunoedited? Front Immunol. 2019;10:1842.

23. Veenhuis RT, et al. Long-term remission despite clonal expansion of replication-competent HIV-1 isolates. JCI Insight. 2018;3(18):122795.

24. Liu J, Roederer M. Differential susceptibility of leukocyte subsets to cytotoxic T cell killing: implications for HIV immunopathogenesis. Cytometry A. 2007;71(2):94-104.

25. Buzon MJ, et al. Susceptibility to CD8 T-cellmediated killing influences the reservoir of latently HIV-1-infected CD4 T cells. J Acquir Immune Defic Syndr. 2014;65(1):1-9.

26. Balaji KN, Schaschke N, Machleidt W, Catalfamo M, Henkart PA. Surface cathepsin B protects cytotoxic lymphocytes from self-destruction after degranulation. JExp Med. 2002;196(4):493-503.

27. Cohnen A, et al. Surface CD107a/LAMP-1 protects natural killer cells from degranulation-associated damage. Blood. 2013;122(8):1411-1418.

28. Clayton KL, et al. Resistance of HIV-infected macrophages to $\mathrm{CD}^{+} \mathrm{T}$ lymphocyte-mediated killing drives activation of the immune system. Nat Immunol. 2018;19(5):475-486.

29. Medema JP, et al. Blockade of the granzyme B/ perforin pathway through overexpression of the serine protease inhibitor PI-9/SPI-6 constitutes a mechanism for immune escape by tumors. Proc Natl Acad Sci U S A. 2001;98(20):11515-11520.

30. Halle S, Halle O, Förster R. Mechanisms and dynamics of T cell-mediated cytotoxicity in vivo. Trends Immunol. 2017;38(6):432-443.

31. Nagata S. Fas ligand-induced apoptosis. Annu Rev Genet. 1999;33:29-55.

32. Nagata S, Golstein P. The Fas death factor. 
Science. 1995;267(5203):1449-1456.

33. Youle RJ, Strasser A. The BCL-2 protein family: opposing activities that mediate cell death. Nat Rev Mol Cell Biol. 2008;9(1):47-59.

34. Adams JM, Cory S. The Bcl-2 protein family: arbiters of cell survival. Science. 1998;281(5381):1322-1326.

35. Hanahan D, Weinberg RA. The hallmarks of cancer. Cell. 2000;100(1):57-70.

36. Charo J, Finkelstein SE, Grewal N, Restifo NP, Robbins PF, Rosenberg SA. Bcl-2 overexpression enhances tumor-specific T-cell survival. Cancer Res. 2005;65(5):2001-2008.

37. Pawlowski J, Kraft AS. Bax-induced apoptotic cell death. Proc Natl Acad Sci U S A. 2000;97(2):529-531.

38. García-Aranda M, Pérez-Ruiz E, Redondo $\mathrm{M}$. Bcl-2 inhibition to overcome resistance to chemo- and immunotherapy. Int J Mol Sci. 2018;19(12):E3950.

39. Souers AJ, et al. ABT-199, a potent and selective BCL-2 inhibitor, achieves antitumor activity while sparing platelets. Nat Med. 2013;19(2):202-208.

40. Roberts AW, et al. Substantial susceptibility of chronic lymphocytic leukemia to BCL2 inhibition: results of a phase I study of navitoclax in patients with relapsed or refractory disease. JClin Oncol. 2012;30(5):488-496.

41. Chomont N, et al. HIV reservoir size and persistence are driven by T cell survival and homeostatic proliferation. Nat Med. 2009;15(8):893-900.

42. Sallusto F, Lenig D, Förster R, Lipp M, Lanzavecchia A. Two subsets of memory T lymphocytes with distinct homing potentials and effector functions. Nature. 1999;401(6754):708-712.

43. Bosque A, Planelles V. Studies of HIV-1 latency in an ex vivo model that uses primary central memory T cells. Methods. 2011;53(1):54-61.

44. Pardons M, et al. Single-cell characterization and quantification of translation-competent viral reservoirs in treated and untreated HIV infection. PLoS Pathog. 2019;15(2):e1007619.

45. Bruner KM, et al. A quantitative approach for measuring the reservoir of latent HIV-1 proviruses. Nature. 2019;566(7742):120-125.

46. Cummins NW, et al. Prime, shock, and kill: priming CD4 T cells from HIV patients with a BCL-2 antagonist before HIV reactivation reduces HIV reservoir size. J Virol. 2016;90(8):4032-4048.

47. Ho YC, et al. Replication-competent noninduced proviruses in the latent reservoir increase barrier to HIV-1 cure. Cell. 2013;155(3):540-551.

48. Bosque A, Planelles V. Induction of HIV-1 latency and reactivation in primary memory $\mathrm{CD} 4+\mathrm{T}$ cells. Blood. 2009;113(1):58-65.

49. Martins LJ, et al. Modeling HIV-1 latency in primary $\mathrm{T}$ cells using a replication-competent virus. AIDS Res Hum Retroviruses. 2016;32(2):187-193.

50. Lam S, et al. Broadly-specific cytotoxic T cells tar- geting multiple HIV antigens are expanded from HIV+ patients: implications for immunotherapy. Mol Ther. 2015;23(2):387-395.

51. Patel S, et al. Functionally active HIV-specific T cells that target Gag and Nef can be expanded from virus-naïve donors and target a range of viral epitopes: implications for a cure strategy after allogeneic hematopoietic stem cell transplantation. Biol Blood Marrow Transplant. 2016;22(3):536-541.

52. Day CL, et al. PD-1 expression on HIVspecific $\mathrm{T}$ cells is associated with T-cell exhaustion and disease progression. Nature. 2006;443(7109):350-354.

53. Jones RB, et al. Tim-3 expression defines a novel population of dysfunctional $\mathrm{T}$ cells with highly elevated frequencies in progressive HIV-1 infection. JExp Med. 2008;205(12):2763-2779.

54. Petrovas C, et al. PD-1 is a regulator of virusspecific $\mathrm{CD} 8+\mathrm{T}$ cell survival in HIV infection. JExp Med. 2006;203(10):2281-2292.

55. Trautmann L, et al. Upregulation of PD-1 expression on HIV-specific CD8+ T cells leads to reversible immune dysfunction. Nat Med. 2006;12(10):1198-1202.

56. Hersperger AR, Migueles SA, Betts MR, Connors M. Qualitative features of the HIV-specific CD8+ T-cell response associated with immunologic control. Curr Opin HIV AIDS. 2011;6(3):169-173.

57. Zaretsky JM, et al. Mutations associated with acquired resistance to PD-1 blockade in melanoma. NEnglJMed.2016;375(9):819-829.

58. Patel SJ, et al. Identification of essential genes for cancer immunotherapy. Nature. 2017;548(7669):537-542.

59. Letai AG. Diagnosing and exploiting cancer's addiction to blocks in apoptosis. Nat Rev Cancer. 2008;8(2):121-132.

60. Deng J, Carlson N, Takeyama K, Dal Cin P, Shipp $\mathrm{M}$, Letai A. BH3 profiling identifies three distinct classes of apoptotic blocks to predict response to ABT-737 and conventional chemotherapeutic agents. Cancer Cell. 2007;12(2):171-185.

61. Del Gaizo Moore V, Brown JR, Certo M, Love TM, Novina CD, Letai A. Chronic lymphocytic leukemia requires BCL2 to sequester prodeath BIM, explaining sensitivity to BCL2 antagonist ABT-737. J Clin Invest. 2007;117(1):112-121.

62. Ito T, Deng X, Carr B, May WS. Bcl-2 phosphorylation required for anti-apoptosis function. J Biol Chem. 1997;272(18):11671-11673.

63. Natesampillai S, et al. HIV protease-generated Casp8p41, when bound and inactivated by $\mathrm{Bcl} 2$, is degraded by the proteasome. JVirol. 2018;92(13):e00037-18.

64. Cummins NW, Sainski-Nguyen AM, Natesampillai S, Aboulnasr F, Kaufmann S, Badley AD. Maintenance of the HIV reservoir is antagonized by selective BCL2 inhibition. J Virol. 2017;91(11):e00012-17.

65. Archin NM, Margolis DM. Emerging strategies to deplete the HIV reservoir. Curr Opin Infect Dis. 2014;27(1):29-35.

66. Perelson AS, Neumann AU, Markowitz M, Leonard JM, Ho DD. HIV-1 dynamics in vivo: virion clearance rate, infected cell life-span, and viral generation time. Science. 1996;271(5255):1582-1586.

67. Wang Z, et al. Expanded cellular clones carrying replication-competent HIV-1 persist, wax, and wane. Proc Natl Acad Sci U S A. 2018;115(11):E2575-E2584.

68. Kuo HH, et al. Anti-apoptotic protein BIRC5 maintains survival of HIV-1-infected $\mathrm{CD} 4^{+} \mathrm{T}$ cells. Immunity. 2018;48(6):1183-1194.e5.

69. Pollack RA, et al. Defective HIV-1 proviruses are expressed and can be recognized by cytotoxic T lymphocytes, which shape the proviral landscape. Cell Host Microbe. 2017;21(4):494-506.e4.

70. Imamichi H, et al. Defective HIV-1 proviruses produce novel protein-coding RNA species in HIV-infected patients on combination antiretroviral therapy. Proc Natl Acad Sci U S A. 2016;113(31):8783-8788.

71. Boidol B, et al. First-in-human response of BCL-2 inhibitor venetoclax in T-cell prolymphocytic leukemia. Blood. 2017;130(23):2499-2503.

72. Seymour JF, et al. Venetoclax-rituximab in relapsed or refractory chronic lymphocytic leukemia. N Engl JMed. 2018;378(12):1107-1120.

73. Mathew R, et al. The BCL-2-selective inhibitor venetoclax spares activated T-cells during anti-tumor immunity. Blood. 2018;132(suppl 1):3704.

74. Haikala HM, et al. Pharmacological reactivation of MYC-dependent apoptosis induces susceptibility to anti-PD-1 immunotherapy. Nat Commun . 2019;10(1):620.

75. Dobin A, et al. STAR: ultrafast universal RNA-seq aligner. Bioinformatics. 2013;29(1):15-21.

76. Trapnell C, Hendrickson DG, Sauvageau M, Goff L, Rinn JL, Pachter L. Differential analysis of gene regulation at transcript resolution with RNA-seq. Nat Biotechnol. 2013;31(1):46-53.

77. Trapnell C, et al. Transcript assembly and quantification by RNA-Seq reveals unannotated transcripts and isoform switching during cell differentiation. Nat Biotechnol. 2010;28(5):511-515.

78. Love MI, Huber W, Anders S. Moderated estimation of fold change and dispersion for RNA-seq data with DESeq2. Genome Biol. 2014;15(12):550.

79. Krämer A, Green G, Pollard J Jr, Tugendreich S. Causal analysis approaches in Ingenuity Pathway Analysis. Bioinformatics. 2014;30(4):523-530.

80. Strain MC, et al. Highly precise measurement of HIV DNA by droplet digital PCR. PLOS ONE. 2013;8(4):e55943.

81. Laird GM, et al. Rapid quantification of the latent reservoir for HIV-1 using a viral outgrowth assay. PLoS Pathog. 2013;9(5):e1003398. 